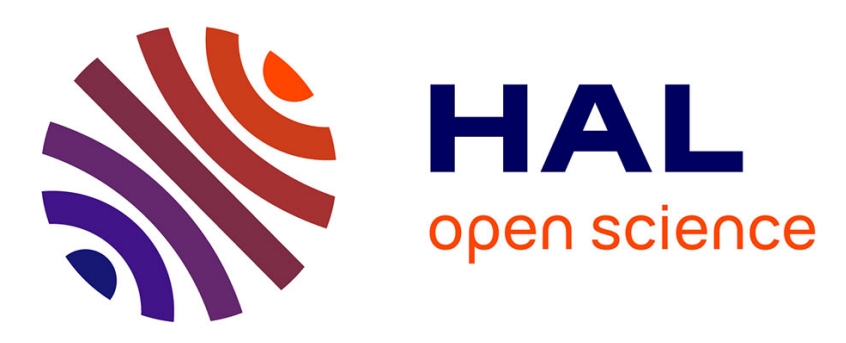

\title{
Reduced models accounting for parallel magnetic perturbations: gyrofluid and FLR-Landau fluid approaches
}

\author{
Emanuele Tassi, Pierre-Louis Sulem, Thierry Passot
}

\section{- To cite this version:}

Emanuele Tassi, Pierre-Louis Sulem, Thierry Passot. Reduced models accounting for parallel magnetic perturbations: gyrofluid and FLR-Landau fluid approaches. Journal of Plasma Physics, 2016, 82 (6), pp.705820601. 10.1017/S0022377816000921 . hal-01434326

\section{HAL Id: hal-01434326 \\ https://hal.science/hal-01434326}

Submitted on 13 Jan 2017

HAL is a multi-disciplinary open access archive for the deposit and dissemination of scientific research documents, whether they are published or not. The documents may come from teaching and research institutions in France or abroad, or from public or private research centers.
L'archive ouverte pluridisciplinaire HAL, est destinée au dépôt et à la diffusion de documents scientifiques de niveau recherche, publiés ou non, émanant des établissements d'enseignement et de recherche français ou étrangers, des laboratoires publics ou privés. 


\title{
Reduced models accounting for parallel magnetic perturbations: gyrofluid and FLR-Landau fluid approaches
}

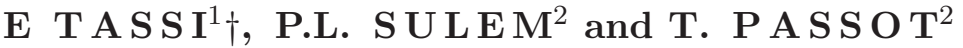 \\ ${ }^{1}$ Aix Marseille Univ, Univ Toulon, CNRS, CPT, Marseille, France \\ ${ }^{2}$ Université Côte d'Azur, CNRS, Observatoire de la Côte d'Azur, Laboratoire J.L. Lagrange,
} Boulevard de l'Observatoire, CS 34229, 06304 Nice Cedex 4, France

(Received ?; revised ?; accepted ?. - To be entered by editorial office)

Reduced models are derived for a strongly magnetized collisionless plasma at scales large relatively to the electron thermal gyroradius, in two asymptotic regimes. One corresponds to cold ions and the other to far sub-ion scales. By including the electron pressure dynamics, these models improve the Hall reduced MHD and the kinetic Alfvén wave model of Boldyrev et al. (2013), respectively. We show that the two models can be obtained either within a gyrofluid formalism (Brizard 1992) or as suitable weakly nonlinear limits of the FLR-Landau fluid of Sulem \& Passot (2015) which extends anisotropic Hallmagnetohydrodynamics by retaining low-frequency kinetic effects. It is noticeable that, at the far sub-ion scales, the simplifications originating from the gyroaveraging operators in the gyrofluid formalism and leading to subdominant ion velocity and temperature fluctuations, correspond, at the level of the FLR-Landau fluid, to cancellation between hydrodynamic contributions and ion finite Larmor radius corrections. Energy conservation properties of the models are discussed and an explicit example of closure relation leading to a model with a Hamiltonian structure is provided.

PACS codes: Authors should not enter PACS codes directly on the manuscript, as these must be chosen during the online submission process and will then be added during the typesetting process (see http://www.aip.org/pacs/ for the full list of PACS codes)

\section{Introduction}

With the aim of describing the dynamics of kinetic Alfvén waves (KAWs) that play an important role in solar wind turbulence, the present paper is devoted to the development of three-dimensional reduced fluid models suitable for finite-beta plasmas, in regimes where magnetic fluctuations along the guide field are non negligible. When concentrating on scales large compared to the ion gyroradius, reduced models were derived from two-fluid equations (Hazeltine et al. 1985; Fitzpatrick \& Porcelli 2004; Schekochihin et al. 2009; Tassi et al. 2010), possibly extended by retaining leading-order finite Larmor radius (FLR) effects (Hsu et al. 1986). Differently, when considering a quasi-transverse collisionless dynamics at scales comparable to or smaller than the ion thermal Larmor radius, gyrofluid models have been constructed by considering the time evolution of a few velocity moments derived from the gyrokinetic equation. As KAWs are low-frequency waves, it seems suitable to turn to gyrofluids. Nevertheless, as most of such models were 
developed for fusion plasmas, they often assume negligible parallel magnetic field fluctuations (as in, e.g., Snyder \& Hammett (2001)). In contrast, spacecraft observations in the solar wind show that parallel and transverse magnetic fluctuations become comparable at small scales (Kiyani et al. 2013). This led us to use as a starting point the gyrofluid model described by Brizard (1992), where parallel magnetic fluctuations are retained. The approximations made in this model for computing finite Larmor radius (FLR) contributions are discussed in Dorland \& Hammett (1993), where it is argued that the effect of gyro-averaging is overestimated, at least at the smallest scales. However, as in the present paper we concentrate on two asymptotic regimes, corresponding to either cold ions or to transverse scales much smaller than the ion thermal gyroradius but much larger than the electron gyroradius, the difference between the gyroaveraging performed by Brizard (1992) or by Dorland \& Hammett (1993) becomes irrelevant.

The present paper is organized as follows. Starting in Section 2 with the gyrofluid model of Brizard (1992), we consider in Section 3 its limiting formulation in the two previously mentioned asymptotic regimes. In the cold-ion regime, the resulting model can be viewed as a Hall reduced MHD model retaining dynamical equations for the parallel and perpendicular electron pressures. The derivation of the sub-ion scale model, on the other hand, provides a systematic basis to the semi-phenomenological model of Boldyrev et al. (2013), in a formulation not limited to isothermal electrons but including a description of the electron pressure dynamics, which thus permits closures of the fluid hierarchy retaining electron Landau damping, as in the approach initiated by Hammett \& Perkins (1990). Section 3 addresses the issue of energy conservation, together with considerations on closure relations ensuring the Hamiltonian character of the system. In Section 4, we construct a weakly-nonlinear version of the FLR-Landau fluid model presented in Sulem \& Passot (2015). In Section 5, we show that the two derived asymptotic gyrofluid models can be recovered from this reduced FLR-Landau fluid, when taking the corresponding limits. It is noticeable that, in contrast with gyrofluids, the FLR-Landau fluid does not involve a drift description and does not prescribe a priori pressure balance. As a consequence, the present analysis provides a bridge between the gyrofluid formalism and a generalized anisotropic Hall-MHD description where low-frequency kinetic effects have been supplemented. Section 6 discusses the spectral validity range of the small-scale model where deviation from kinetic theory can be mainly attributed to the neglect of electron inertia. We conclude in Section 7.

\section{The gyrofluid model}

We consider as starting point a simplified version of the gyrokinetic equation presented in Brizard $(1989,1992)$, where background magnetic inhomogeneities and curvature have been neglected. It reads

$$
\begin{aligned}
& \frac{\partial F_{\alpha}}{\partial t}+\frac{c}{B}\left[J_{0} \delta \phi-\frac{v}{c} J_{0} \delta A_{\|}+\frac{\mu_{\alpha}}{q_{\alpha}} \frac{2}{k_{\perp} \rho_{\perp_{\alpha}}} J_{1} \delta B_{z}, F_{\alpha}\right]+v \frac{\partial F_{\alpha}}{\partial z} \\
& -\left(\frac{q_{\alpha}}{m_{\alpha} c} \frac{\partial}{\partial t} J_{0} \delta A_{\|}+\frac{q_{\alpha}}{m_{\alpha}} \frac{\partial}{\partial z} J_{0} \delta \phi+\frac{\mu_{\alpha}}{m_{\alpha}} \frac{2}{k_{\perp} \rho_{\perp_{\alpha}}} \frac{\partial}{\partial z} J_{1} \delta B_{z}-\frac{q_{\alpha}}{m_{\alpha} B}\left[J_{0} \delta A_{\|}, J_{0} \delta \phi\right]\right. \\
& \left.-\frac{\mu_{\alpha}}{m_{\alpha} B}\left[J_{0} \delta A_{\|}, \frac{2}{k_{\perp} \rho_{\perp \alpha}} J_{1} \delta B_{z}\right]\right) \frac{\partial F_{\alpha}}{\partial v}=0,
\end{aligned}
$$

where $F_{\alpha}\left(x, y, z, v, \mu_{\alpha}, t\right)$ is the gyrocenter distribution function for the particle species $\alpha$. For simplicity, we consider a plasma composed of electrons and by one single ionized ion species, permeated by a constant magnetic guide field. The index $\alpha$ will then take the 
values $i$ for ions and $e$ for electrons. In particular, one has $q_{i}=e$ and $q_{e}=-e$ to indicate the charge of the ions and of the electrons, respectively. On the other hand, we denote as $m_{i}=M$ and $m_{e}=m$ the ion and electron mass, respectively. The spatial Cartesian coordinates $x, y, z$ span a domain $\left[0, L_{x}\right] \times\left[0, L_{y}\right] \times\left[0, L_{z}\right]$ where we impose periodic boundary conditions, whereas $v \in \mathbb{R}, \mu_{\alpha} \in \mathbb{R}, t \in \mathbb{R}$ with $t \geqslant 0$ and $\mu_{\alpha} \geqslant 0$. We denote as $\mathbf{k}=\mathbf{k}_{\perp}+\mathbf{k}_{\|}$the wave vector of a generic field in Fourier space, with $\mathbf{k}_{\perp}=k_{x} \hat{\mathbf{x}}+k_{y} \hat{\mathbf{y}}$ and $\mathbf{k}_{\|}=k_{z} \hat{\mathbf{z}}$ indicating the components perpendicular and parallel to the strong background magnetic guide field directed along the $z$ axis. Based on the strong guide field ordering, we assume $k_{z} / k_{\perp} \sim \delta B_{z} / B_{0} \sim \epsilon \ll 1$, with $k_{\perp}$ the modulus of the perpendicular wave vector, $B_{0}$ the (constant) amplitude of the strong guide field component and $\delta B_{z}$ the perturbation along the guide field direction. With such assumptions, the expression for the magnetic field (up to terms of order $\epsilon^{2}$ ) is given by $\mathbf{B}(x, y, z, t)=\nabla \delta A_{\|}(x, y, z, t) \times \hat{\mathbf{z}}+$ $\left(B_{0}+\delta B_{z}(x, y, z, t)\right) \hat{\mathbf{z}}$. The field $\delta \phi(x, y, z, t)$, on the other hand, denotes the electrostatic potential.The coordinate $v$ represents the velocity along the $z$ direction. The expression for the magnetic moment $\mu_{\alpha}$, on the other hand, is given, to lowest order, by $\mu_{\alpha}=$ $m_{\alpha} v_{\perp}^{2} / 2 B_{0}$, with $v_{\perp}$ indicating the modulus of the perpendicular velocity coordinate. The constant $c$ refers to the speed of light, whereas $J_{0}$ and $J_{1}$ are operators that, in Fourier space, correspond to the multiplicative factors $J_{0}\left(k_{\perp} \rho_{\perp_{\alpha}}\right)$ and $J_{1}\left(k_{\perp} \rho_{\perp_{\alpha}}\right)$, with $J_{0}$ and $J_{1}$ denoting the zeroth and first order Bessel functions of the first kind. Here, $\rho_{\perp \alpha}=v_{\perp} / \Omega_{\alpha}$ and $\Omega_{\alpha}=\left|q_{\alpha}\right| B_{0} /\left(m_{\alpha} c\right)$ are the Larmor radius and the cyclotron frequency associated with the species $\alpha$, respectively. Finally, the canonical bracket of two functions $f$ and $g$ is defined as $[f, g]=\partial_{x} f \partial_{y} g-\partial_{y} f \partial_{x} g$.

The distribution function of each gyrocenter species is assumed to be given by the sum

$$
F_{\alpha}\left(x, y, z, v, \mu_{\alpha}, t\right)=\mathcal{F}_{e q_{\alpha}}\left(v, \mu_{\alpha}\right)+\tilde{f}_{\alpha}\left(x, y, z, v, \mu_{\alpha}, t\right),
$$

of an equilibrium Maxwellian

$$
\mathcal{F}_{e q_{\alpha}}\left(v, \mu_{\alpha}\right)=n_{0}\left(\frac{m_{\alpha}}{2 \pi T_{\alpha}}\right)^{3 / 2} \exp \left(-\frac{m_{\alpha} v^{2}}{2 T_{\alpha}}-\frac{\mu_{\alpha} B_{0}}{T_{\alpha}}\right)
$$

where $n_{0}$ is the uniform equilibrium density, and a perturbation $\tilde{f}_{\alpha}$ so that $\tilde{f}_{\alpha} / \mathcal{F}_{e q_{\alpha}}=$ $\mathcal{O}(\epsilon)$.

The scalar electromagnetic fields $\delta \phi, \delta A_{\|}$and $\delta B_{z}$ are related to the perturbed distribution functions $\widetilde{f}_{\alpha}$ by means of Poisson's equation

$$
\nabla_{\perp}^{2} \delta \phi=-4 \pi \sum_{\alpha=e, i} q_{\alpha} \int d \mathcal{W}_{\alpha}\left(J_{0} \widetilde{f}_{\alpha}+\left(J_{0}^{2}-1\right) \mathcal{F}_{e q_{\alpha}} \frac{q_{\alpha} \delta \phi}{T_{\alpha}}+\frac{\mu_{\alpha} B_{0}}{T_{\alpha}} J_{0} \frac{2 J_{1}}{k_{\perp} \rho_{\perp \alpha}} \mathcal{F}_{e q_{\alpha}} \frac{\delta B_{z}}{B_{0}}\right)
$$

and of the parallel and perpendicular components of Ampère's law

$$
\nabla_{\perp}^{2} \delta A_{\|}=-\frac{4 \pi}{c} \sum_{\alpha=e, i} q_{\alpha} \int d \mathcal{W}_{\alpha} v J_{0} \widetilde{f}_{\alpha}
$$

and

$$
\begin{aligned}
\nabla_{\perp} \delta B_{z} \times \widehat{\mathbf{z}}= & 4 \pi \widehat{\mathbf{z}} \times \sum_{\alpha=e, i} \int d \mathcal{W}_{\alpha}\left(\mu_{\alpha} \frac{2 J_{1}}{k_{\perp} \rho_{\perp \alpha}} \nabla_{\perp} \widetilde{f}_{\alpha}+\mu_{\alpha} J_{0} \frac{2 J_{1}}{k_{\perp} \rho_{\perp \alpha}} \mathcal{F}_{e q_{\alpha}} \nabla_{\perp} \frac{q_{\alpha} \delta \phi}{T_{\alpha}}\right. \\
& \left.+\frac{\mu_{\alpha}^{2} B}{T_{\alpha}}\left(\frac{2 J_{1}}{k_{\perp} \rho_{\perp \alpha}}\right)^{2} \mathcal{F}_{e q_{\alpha}} \nabla_{\perp} \frac{\delta B_{z}}{B_{0}}\right) .
\end{aligned}
$$

We then introduce the following gyrofluid moments built from the perturbation of the 
distribution function:

$$
\begin{aligned}
& \widetilde{N}_{\alpha}=\int d \mathcal{W}_{\alpha} \widetilde{f}_{\alpha}, \quad n_{0} \widetilde{U}_{\alpha}=\int d \mathcal{W}_{\alpha} v \widetilde{f}_{\alpha} \\
& \widetilde{P}_{\|_{\alpha}}=m_{\alpha} \int d \mathcal{W}_{\alpha} v^{2} \widetilde{f}_{\alpha}, \quad \widetilde{P}_{\perp_{\alpha}}=\int d \mathcal{W}_{\alpha} \mu_{\alpha} B_{0} \widetilde{f}_{\alpha} \\
& \widetilde{Q}_{\|_{\alpha}}=m_{\alpha} \int d \mathcal{W}_{\alpha} v^{3} \widetilde{f}_{\alpha}-3 n_{0} T_{\alpha} \widetilde{U}_{\alpha}, \quad \widetilde{Q}_{\perp_{\alpha}}=\int d \mathcal{W}_{\alpha} \mu_{\alpha} B_{0} v \widetilde{f}_{\alpha}-n_{0} T_{\alpha} \widetilde{U}_{\alpha} \\
& m_{\alpha} \widetilde{R}_{\| \perp \alpha}=m_{\alpha} \int d \mathcal{W}_{\alpha} v^{2} \mu_{\alpha} B_{0} \widetilde{f}_{\alpha}-T_{\alpha} \widetilde{P}_{\|_{\alpha}}-T_{\alpha} \widetilde{P}_{\perp_{\alpha}}+T_{\alpha}^{2} \widetilde{N}_{\alpha} \\
& m_{\alpha} \widetilde{R}_{\perp \perp \alpha}=\frac{1}{2} \int d \mathcal{W}_{\alpha} \mu_{\alpha}^{2} B_{0}^{2} \widetilde{f}_{\alpha}-2 T_{\alpha} \widetilde{P}_{\perp_{\alpha}}+T_{\alpha}^{2} \widetilde{N}_{\alpha}
\end{aligned}
$$

with the volume element $d \mathcal{W}_{\alpha}$ defined by $d \mathcal{W}_{\alpha}=\left(2 \pi B_{0} / m_{\alpha}\right) d \mu_{\alpha} d v$. In Eqs. (2.7)-(2.11), $\widetilde{N}_{\alpha}$ and $\widetilde{U}_{\alpha}$ indicate the gyrofluid density and parallel velocity, $\widetilde{P}_{\|_{\alpha}}$ and $\widetilde{P}_{\perp_{\alpha}}$ the parallel and perpendicular pressure, $\widetilde{Q}_{\|_{\alpha}}$ and $\widetilde{Q}_{\perp_{\alpha}}$ the parallel and perpendicular components of the parallel heat fluxes, and $\widetilde{R}_{\| \perp \alpha}$ and $\widetilde{R}_{\perp \perp \alpha}$ the perpendicular component of the parallel and perpendicular energy-weighted pressure tensors, respectively. Furthermore, $n_{0}$ and $T_{\alpha}$ are the equilibrium density and temperature (assumed isotropic) of particles of species $\alpha$, respectively.

We then define the following non-dimensional quantities (denoted with overbars):

$$
\begin{aligned}
& \bar{t}=\Omega_{i} t, \quad \bar{x}=\frac{x}{\rho_{s}}, \quad \bar{y}=\frac{y}{\rho_{s}}, \quad \bar{z}=\frac{z}{\rho_{s}}, \quad \bar{k}_{\perp}=k_{\perp} \rho_{s}, \quad \bar{k}_{z}=k_{z} \rho_{s}, \quad \Delta_{s}=\frac{\rho_{s}^{2}}{2} \nabla_{\perp}^{2}, \\
& \bar{N}_{\alpha}=\frac{\widetilde{N}_{\alpha}}{n_{0}}, \quad \bar{U}_{\alpha}=\frac{\widetilde{U}_{\alpha}}{c_{s}}, \quad \bar{P}_{\|_{\alpha}}=\frac{\widetilde{P}_{\|_{\alpha}}}{n_{0} T_{\alpha}}, \quad \bar{P}_{\perp_{\alpha}}=\frac{\widetilde{P}_{\perp_{\alpha}}}{n_{0} T_{\alpha}}, \\
& \bar{Q}_{\|_{\alpha}}=\frac{\widetilde{Q}_{\|_{\alpha}}}{n_{0} T_{\alpha} c_{s}}, \quad \bar{Q}_{\perp_{\alpha}}=\frac{\widetilde{Q}_{\perp_{\alpha}}}{n_{0} T_{\alpha} c_{s}}, \quad \bar{R}_{x_{\alpha}}=m_{\alpha} \frac{\widetilde{R}_{\| \perp \alpha}}{n_{0} T_{\alpha}^{2}}, \quad \bar{R}_{\perp_{\alpha}}^{(\perp)}=m_{\alpha} \frac{\widetilde{R}_{\perp \perp \alpha}}{n_{0} T_{\alpha}^{2}}, \\
& \varphi=\frac{e \delta \phi}{T_{e}}, \quad A_{\|}=\frac{\delta A_{\|}}{B_{0} \rho_{s}}, \quad B_{z}=\frac{\delta B_{z}}{B_{0}} .
\end{aligned}
$$

We introduce the non-dimensional parameters:

$$
\tau=\frac{T_{i}}{T_{e}}, \quad \delta=\sqrt{\frac{m}{M}}, \quad \beta_{\alpha}=8 \pi \frac{n_{0} T_{\alpha}}{B_{0}^{2}},
$$

and assume the ordering

$$
\begin{aligned}
& \bar{x} \sim \bar{y} \sim \bar{z} \sim \bar{k}_{\perp} \sim \beta_{e}=\mathcal{O}(1), \\
& \bar{k}_{\|} \sim \frac{1}{\bar{t}} \sim \bar{N}_{\alpha} \sim \bar{U}_{\alpha} \sim \bar{P}_{\|_{\alpha}} \sim \bar{P}_{\perp_{\alpha}} \sim \bar{Q}_{\|_{\alpha}} \sim \bar{Q}_{\perp_{\alpha}} \sim \bar{R}_{x_{\alpha}} \sim \bar{R}_{\perp_{\alpha}}^{(\perp)} \\
& \sim \varphi \sim A_{\|} \sim B_{z}=\mathcal{O}(\epsilon) \ll 1
\end{aligned}
$$

where $c_{s}=\sqrt{T_{e} / M}$ is the sound speed and $\rho_{s}=c_{s} / \Omega_{i}$ the sonic Larmor radius. Note that the normalization and the ordering differ from those adopted in Brizard (1992).

Taking moments of Eq.(2.1) leads to the set of normalized evolution equations (where 
the overbars were removed from the non-dimensional quantities)

$$
\begin{aligned}
& \frac{\partial N_{e}}{\partial t}+\left[\mathrm{e}^{\delta^{2} \Delta_{s}} \varphi, N_{e}\right]+\delta^{2}\left[\Delta_{s} \mathrm{e}^{\delta^{2} \Delta_{s}} \varphi, P_{\perp_{e}}-N_{e}\right]-\left[\mathrm{e}^{\delta^{2} \Delta_{s}} A_{\|}, U_{e}\right]-\left[\mathrm{e}^{\delta^{2} \Delta_{s}} B_{z}, P_{\perp_{e}}\right] \\
& -\delta^{2}\left[\Delta_{s} \mathrm{e}^{\delta^{2} \Delta_{s}} B_{z}, P_{\perp_{e}}-N_{e}\right]+\frac{\partial U_{e}}{\partial z}=0, \\
& \frac{\partial}{\partial t}\left(\delta^{2} U_{e}-\mathrm{e}^{\delta^{2} \Delta_{s}} A_{\|}\right)+\delta^{2}\left[\mathrm{e}^{\delta^{2} \Delta_{s}} \varphi, U_{e}\right]-\left[\mathrm{e}^{\delta^{2} \Delta_{s}} A_{\|}, P_{\|_{e}}\right]-\delta^{2}\left[\Delta_{s} \mathrm{e}^{\delta^{2} \Delta_{s}} A_{\|}, P_{\perp_{e}}-N_{e}\right] \\
& -\delta^{2}\left[\mathrm{e}^{\delta^{2} \Delta_{s}} B_{z}, U_{e}\right]-\delta^{2}\left[B_{z}, Q_{\perp_{e}}\right]-\Gamma_{0}\left(\delta^{2} \Delta_{s}^{\varphi}, \delta^{2} \Delta_{s}^{A}\right)\left[\varphi, A_{\|}\right] \\
& +\left(\Gamma_{0}\left(\delta^{2} \Delta_{s}^{B}, \delta^{2} \Delta_{s}^{A}\right)+\delta^{2} \Delta_{s} \Gamma_{1}\left(\delta^{2} \Delta_{s}^{B}, \delta^{2} \Delta_{s}^{A}\right)\right)\left[B_{z}, A_{\|}\right] \\
& +\frac{\partial}{\partial z}\left(P_{\|_{e}}-\mathrm{e}^{\delta^{2} \Delta_{s}} \varphi+\mathrm{e}^{\delta^{2} \Delta_{s}} B_{z}\right)=0 \\
& \frac{\partial P_{\|_{e}}}{\partial t}+\left[\mathrm{e}^{\delta^{2} \Delta_{s}} \varphi, P_{\|_{e}}\right]+\delta^{2}\left[\Delta_{s} \mathrm{e}^{\delta^{2} \Delta_{s}} \varphi, P_{\perp_{e}}-N_{e}\right]-\left[A_{\|}, Q_{\|_{e}}\right]-3\left[\mathrm{e}^{\delta^{2} \Delta_{s}} A_{\|}, U_{e}\right] \\
& -\left[\mathrm{e}^{\delta^{2} \Delta_{s}} B_{z}, P_{\|_{e}}+P_{\perp_{e}}-N_{e}\right]-\delta^{2}\left[\Delta_{s} \mathrm{e}^{\delta^{2} \Delta_{s}} B_{z}, P_{\perp_{e}}-N_{e}\right]-\left[B_{z}, R_{\| \perp_{e}}\right] \\
& +\frac{\partial}{\partial z}\left(Q_{\|_{e}}+3 U_{e}\right)=0 \\
& \frac{\partial P_{\perp_{e}}}{\partial t}+\left[\left(1+\delta^{2} \Delta_{s}\right) \mathrm{e}^{\delta^{2} \Delta_{s}} \varphi, P_{\perp_{e}}\right]+\delta^{2}\left[\Delta_{s}\left(2+\delta^{2} \Delta_{s}\right) \mathrm{e}^{\delta^{2} \Delta_{s}} \varphi, P_{\perp_{e}}-N_{e}\right]-\left[\mathrm{e}^{\delta^{2} \Delta_{s}} A_{\|}, U_{e}\right] \\
& -\left[A_{\|}, Q_{\perp_{e}}\right]-\left[\left(2+\delta^{2} \Delta_{s}\right) \mathrm{e}^{\delta^{2} \Delta_{s}} B_{z}, 2 P_{\perp_{e}}-N_{e}\right]-\delta^{2}\left[\Delta_{s}\left(3+\delta^{2} \Delta_{s}\right) \mathrm{e}^{\delta^{2} \Delta_{s}} B_{z}, P_{\perp_{e}}-N_{e}\right] \\
& -2\left[B_{z}, R_{\perp \perp e}\right]+\frac{\partial}{\partial z}\left(U_{e}+Q_{\perp_{e}}\right)=0, \\
& \frac{\partial N_{i}}{\partial t}+\left[\mathrm{e}^{\tau \Delta_{s}} \varphi, N_{i}\right]+\tau\left[\Delta_{s} \mathrm{e}^{\tau \Delta_{s}} \varphi, P_{\perp_{i}}-N_{i}\right]-\left[\mathrm{e}^{\tau \Delta_{s}} A_{\|}, U_{i}\right]+\tau\left[\mathrm{e}^{\tau \Delta_{s}} B_{z}, P_{\perp_{i}}\right] \\
& +\tau\left[\Delta_{s} \mathrm{e}^{\tau \Delta_{s}} B_{z}, P_{\perp_{i}}-N_{i}\right]+\frac{\partial U_{i}}{\partial z}=0 \\
& \frac{\partial}{\partial t}\left(U_{i}+\mathrm{e}^{\tau \Delta_{s}} A_{\|}\right)+\left[\mathrm{e}^{\tau \Delta_{s}} \varphi, U_{i}\right]-\tau\left[\mathrm{e}^{\tau \Delta_{s}} A_{\|}, P_{\|_{i}}\right]-\tau\left[\Delta_{s} \mathrm{e}^{\tau \Delta_{s}} A_{\|}, P_{\perp_{i}}-N_{i}\right] \\
& +\tau\left[\mathrm{e}^{\tau \Delta_{s}} B_{z}, U_{i}\right]+\tau\left[B_{z}, Q_{\perp_{i}}\right]+\Gamma_{0}\left(\tau \Delta_{s}^{\varphi}, \tau \Delta_{s}^{A}\right)\left[\varphi, A_{\|}\right] \\
& +\tau\left(\Gamma_{0}\left(\tau \Delta_{s}^{B}, \tau \Delta_{s}^{A}\right)+\tau \Delta_{s} \Gamma_{1}\left(\delta^{2} \Delta_{s}^{B}, \delta^{2} \Delta_{s}^{A}\right)\right)\left[B_{z}, A_{\|}\right]+\frac{\partial}{\partial z}\left(\tau P_{\|_{i}}+\mathrm{e}^{\tau \Delta_{s}} \varphi+\mathrm{e}^{\tau \Delta_{s}} B_{z}\right)=0, \\
& \frac{\partial P_{\|_{i}}}{\partial t}+\left[\mathrm{e}^{\tau \Delta_{s}} \varphi, P_{\|_{i}}\right]+\tau\left[\Delta_{s} \mathrm{e}^{\tau \Delta_{s}} \varphi, P_{\perp_{i}}-N_{i}\right]-\left[A_{\|}, Q_{\|_{i}}\right]-3\left[\mathrm{e}^{\tau \Delta_{s}} A_{\|}, U_{i}\right] \\
& +\tau\left[\mathrm{e}^{\tau \Delta_{s}} B_{z}, P_{\|_{i}}+P_{\perp_{i}}-N_{i}\right]+\tau^{2}\left[\Delta_{s} \mathrm{e}^{\tau \Delta_{s}} B_{z}, P_{\perp_{i}}-N_{i}\right] \\
& +\tau\left[B_{z}, R_{\| \perp i}\right]+\frac{\partial}{\partial z}\left(Q_{\|_{i}}+3 U_{i}\right)=0, \\
& \frac{\partial P_{\perp_{i}}}{\partial t}+\left[\left(1+\tau \Delta_{s}\right) \mathrm{e}^{\tau \Delta_{s}} \varphi, P_{\perp_{i}}\right]+\tau\left[\Delta_{s}\left(2+\tau \Delta_{s}\right) \mathrm{e}^{\tau \Delta_{s}} \varphi, P_{\perp_{i}}-N_{i}\right]-\left[\mathrm{e}^{\tau \Delta_{s}} A_{\|}, U_{i}\right] \\
& -\left[A_{\|}, Q_{\perp_{i}}\right]+\tau\left[\left(2+\tau \Delta_{s}\right) \mathrm{e}^{\tau \Delta_{s}} B_{z}, 2 P_{\perp_{i}}-N_{i}\right]+\tau\left[\Delta_{s}\left(3+\tau \Delta_{s}\right) \mathrm{e}^{\tau \Delta_{s}} B_{z}, P_{\perp_{i}}-N_{i}\right] \\
& +2 \tau\left[B_{z}, R_{\perp \perp i}\right]+\frac{\partial}{\partial z}\left(U_{i}+Q_{\perp_{i}}\right)=0 .
\end{aligned}
$$

for the first four electron and ion gyrofluid moments, where terms of order up to $\epsilon^{2}$ have been retained, while FLR effects have been estimated only up to leading order in the terms involving heat fluxes and energy-weighted pressures. The operators $\Gamma_{0}$ and $\Gamma_{1}$ are defined as $\Gamma_{0}\left(z, z^{\prime}\right)=I_{0}\left(z z^{\prime}\right) \exp \left(z+z^{\prime}\right)$ and $\Gamma_{1}\left(z, z^{\prime}\right)=I_{1}\left(z z^{\prime}\right) \exp \left(z+z^{\prime}\right)$, where $I_{n}$ 
is the modified Bessel function of the first kind of order $n$. Furthermore, the symbols $\Delta_{s}^{B}, \Delta_{s}^{A}$ and $\Delta_{s}^{\varphi}$ refer to the Laplacian operator $\Delta_{s}$ acting on $B_{z}, A_{\|}$and $\varphi$, respectively. Equations (2.15)-(2.22) are supplemented by Poisson's equation

$$
\begin{aligned}
& \frac{v_{A}^{2}}{c^{2}} \nabla_{\perp}^{2} \varphi=\mathrm{e}^{\delta^{2} \Delta_{s}} N_{e}+\delta^{2} \Delta_{s} \mathrm{e}^{\delta^{2} \Delta_{s}}\left(P_{\perp_{e}}-N_{e}\right)-\left(I_{0}\left(2 \delta^{2} \Delta_{s}\right)-1\right) \mathrm{e}^{2 \delta^{2} \Delta_{s}} \varphi \\
& +\left(I_{0}\left(2 \delta^{2} \Delta_{s}\right)+I_{1}\left(2 \delta^{2} \Delta_{s}\right)\right) \mathrm{e}^{2 \delta^{2} \Delta_{s}} B_{z}-\mathrm{e}^{\tau \Delta_{s}} N_{i}-\tau \Delta_{s} \mathrm{e}^{\tau \Delta_{s}}\left(P_{\perp_{i}}-N_{i}\right) \\
& -\left(I_{0}\left(2 \tau \Delta_{s}\right) \mathrm{e}^{2 \tau \Delta_{s}}-1\right) \frac{\varphi}{\tau}-\left(I_{0}\left(2 \tau \Delta_{s}\right)+I_{1}\left(2 \tau \Delta_{s}\right)\right) \mathrm{e}^{2 \tau \Delta_{s}} B_{z}
\end{aligned}
$$

where $v_{A}=B_{0} /\left(4 \pi M n_{0}\right)^{1 / 2}$ is the Alfvén speed at equilibrium, and by the parallel and perpendicular components of Ampère's law which yield

$$
\nabla_{\perp}^{2} A_{\|}=\frac{\beta_{e}}{2}\left(\mathrm{e}^{\delta^{2} \Delta_{s}} U_{e}-\mathrm{e}^{\tau \Delta_{s}} U_{i}\right),
$$

and

$$
\begin{aligned}
& B_{z}=-\frac{\beta_{e}}{2}\left(\mathrm{e}^{\delta^{2} \Delta_{s}} P_{\perp_{e}}+\delta^{2} \Delta_{s} \mathrm{e}^{\delta^{2} \Delta_{s}}\left(P_{\perp_{e}}-N_{e}\right)-\left(I_{0}\left(2 \delta^{2} \Delta_{s}\right)+I_{1}\left(2 \delta^{2} \Delta_{s}\right)\right) \mathrm{e}^{2 \delta^{2} \Delta_{s}} \varphi\right. \\
& +2\left(I_{0}\left(2 \delta^{2} \Delta_{s}\right)+I_{1}\left(2 \delta^{2} \Delta_{s}\right)\right) \mathrm{e}^{2 \delta^{2} \Delta_{s}} B_{z}+\tau \mathrm{e}^{\tau \Delta_{s}} P_{\perp_{i}}+\tau^{2} \Delta_{s} \mathrm{e}^{\tau \Delta_{s}}\left(P_{\perp_{i}}-N_{i}\right) \\
& \left.+\left(I_{0}\left(2 \tau \Delta_{s}\right)+I_{1}\left(2 \tau \Delta_{s}\right)\right) \mathrm{e}^{2 \tau \Delta_{s}} \varphi+2 \tau\left(I_{0}\left(2 \tau \Delta_{s}\right)+I_{1}\left(2 \tau \Delta_{s}\right)\right) \mathrm{e}^{2 \tau \Delta_{s}} B_{z}\right)
\end{aligned}
$$

Equations (2.23), (2.24) and (2.25), obtained from Eqs. (2.4), (2.5) and (2.6) respectively, provide relations that permit the fields $\varphi, A_{\|}$and $B_{z}$ to be expressed in terms of the eight gyrofluid moments $N_{e}, N_{i}, U_{e}, U_{i}, P_{\|_{e}}, P_{\|_{i}}, P_{\perp_{e}}$ and $P_{\perp_{i}}$.

\section{Two asymptotic regimes}

The complexity of the gyrofluid model discussed above suggests to pay attention to two asymptotic regimes where drastic simplifications take place in the model formulation.

\subsection{The cold-ion limit}

We here consider the limit of negligible electron inertia $(\delta \ll 1)$, cold ions $(\tau \ll 1)$ and non-relativistic Alfvén speed $\left(v_{A} / c \ll 1\right)$. Introducing the constant $b=\left(\beta_{e} / 2\right)\left(1+\beta_{e}\right)^{-1}$ and the parallel gradient operator

$$
\nabla_{\|}=\frac{\partial}{\partial z}-\left[A_{\|}, \cdot\right]
$$


Eqs. (2.15)-(2.22), (2.23), (2.24) and (2.25) become

$$
\begin{aligned}
& \frac{\partial N_{e}}{\partial t}+\left[\varphi, N_{e}\right]-\left[B_{z}, P_{\perp_{e}}\right]+\nabla_{\|} U_{e}=0 \\
& \frac{\partial A_{\|}}{\partial t}-\nabla_{\|}\left(P_{\|_{e}}-\varphi+B_{z}\right)=0 \\
& \frac{\partial P_{\|_{e}}}{\partial t}+\left[\varphi, P_{\|_{e}}\right]-\left[B_{z}, P_{\|_{e}}\right]-\left[B_{z}, P_{\perp_{e}}\right]+\left[B_{z}, N_{e}\right]-\left[B_{z}, R_{\| \perp_{e}}\right]+\nabla_{\|}\left(Q_{\|_{e}}+3 U_{e}\right)=0 \\
& \frac{\partial P_{\perp_{e}}}{\partial t}+\left[\varphi, P_{\perp_{e}}\right]-4\left[B_{z}, P_{\perp_{e}}\right]+2\left[B_{z}, N_{e}\right]-2\left[B_{z}, R_{\perp \perp e}\right]+\nabla_{\|}\left(U_{e}+Q_{\perp_{e}}\right)=0 \\
& \frac{\partial N_{i}}{\partial t}+\left[\varphi, N_{i}\right]+\nabla_{\|} U_{i}=0 \\
& \frac{\partial}{\partial t}\left(U_{i}+A_{\|}\right)+\left[\varphi, U_{i}\right]+\nabla_{\|} \varphi=0, \\
& \frac{\partial P_{\|_{i}}}{\partial t}+\left[\varphi, P_{\|_{i}}\right]+\nabla_{\|}\left(Q_{\|_{i}}+3 U_{i}\right)=0 \\
& \frac{\partial P_{\perp_{i}}}{\partial t}+\left[\varphi, P_{\perp_{i}}\right]+\nabla_{\|}\left(U_{i}+Q_{\perp_{i}}\right)=0 \\
& \nabla_{\perp}^{2} \varphi=N_{e}-N_{i}, \\
& \nabla_{\perp}^{2} A_{\|}=\frac{\beta_{e}}{2}\left(U_{e}-U_{i}\right) \\
& B_{z}=-b P_{\perp_{e}} .
\end{aligned}
$$

Provided that closures for heat fluxes and fourth order moments are given, the system (3.2)-(3.12) yields a model for finite electron beta plasmas, accounting for parallel magnetic perturbations and valid at scales larger than the electron thermal gyroradius, in the limit of low ion temperature.

\subsection{Sub-ion scale limit}

We consider in this section a different situation, where we still have $v_{A} / c \ll 1$ and $2 \delta^{2} \Delta_{s}=\rho_{e}^{2} \nabla_{\perp}^{2} \ll 1$, with $\rho_{e}$ indicating the electron thermal gyroradius, but where scales are now much smaller than the ion thermal radius $\rho_{i}$, i.e. $2 \tau \Delta_{s}=\rho_{i}^{2} \nabla_{\perp}^{2} \gg 1$. In order to derive a model in this sub-ion scale limit, it is convenient to introduce an additional dimensionless parameter $\mu \ll 1$. We then impose the following subsidiary ordering between the non-dimensional quantities:

$$
\begin{aligned}
& \frac{\partial}{\partial x} \sim \frac{\partial}{\partial y}=\mathcal{O}(1 / \mu), \\
& \tau \sim \beta_{e}=\mathcal{O}(1), \\
& \frac{\partial}{\partial t}=\mathcal{O}(\epsilon / \mu), \\
& \frac{\partial}{\partial z} \sim U_{\alpha} \sim Q_{\perp_{\alpha}} \sim Q_{\|_{\alpha}}=\mathcal{O}(\epsilon), \\
& N_{\alpha} \sim P_{\|_{\alpha}} \sim P_{\perp_{\alpha}} \sim R_{x_{\alpha}} \sim R_{\perp_{\alpha}}^{(\perp)} \sim \varphi \sim B_{z}=\mathcal{O}(\epsilon \mu), \\
& A_{\|}=\mathcal{O}\left(\epsilon \mu^{2}\right) .
\end{aligned}
$$

As a result of such an ordering and of the assumption $2 \delta^{2} \Delta_{s} \ll 1$, one still obtains from Eqs. (2.15)-(2.18) the evolution equations (3.2)-(3.5) for the four electron moments, as 
in Sec. 3.1. On the other hand, in the sub-ion scale limit, Poisson's equation (2.23) yields

$$
\varphi=-\tau N_{e}-\tau B_{z}
$$

whereas the parallel and perpendicular Ampère's law (2.24) and (2.25) give

$$
\nabla_{\perp}^{2} A_{\|}=\frac{\beta_{e}}{2} U_{e}
$$

and

$$
B_{z}=b\left(\varphi-P_{\perp_{e}}\right)
$$

respectively.

From Eqs. (3.14) and (3.16) one obtains the relations

$$
\varphi=-\frac{\tau}{1+\tau b} N_{e}+\frac{\tau b}{1+\tau b} P_{\perp_{e}}, \quad B_{z}=-\frac{\tau b}{1+\tau b} N_{e}-\frac{b}{1+\tau b} P_{\perp_{e}}
$$

which, together with Eq. (3.15), permit, in this regime, electromagnetic fluctuations to be expressed in terms of gyrofluid moments. Note that evolution equations for the ion moments do not enter this model.

Thus, Eqs. (3.2)-(3.5), together with Eqs. (3.15) and (3.17), yield a set of equations applicable to plasmas with finite beta for both ions and electrons, accounting for parallel magnetic perturbations and valid on scales larger than the electron thermal gyroradius but smaller than the ion thermal gyroradius.

\subsection{Energy conservation}

The two asymptotic models derived in the previous subsections are not closed because expressions for the electron and ion heat fluxes $Q_{\|_{e}}, Q_{\perp_{e}}, Q_{\|_{i}}, Q_{\perp_{i}}$ as well as for the electron energy-weighted pressure tensor $R_{\| \perp_{e}}$ and $R_{\perp \perp e}$, in terms of lower order moments, are not provided. Because no dissipative effect has been included in the derivation of the models, it is important to verify that, unless dissipation is introduced through closure relations on the heat fluxes and energy-weighted pressure tensors, both systems possess conserved energy functionals. For this purpose, it is convenient to rewrite the two models replacing the pressures in favor of the parallel and perpendicular temperature fluctuations, defined as $T_{\|_{\alpha}}=P_{\|_{\alpha}}-N_{\alpha}$ and $T_{\perp_{\alpha}}=P_{\perp_{\alpha}}-N_{\alpha}$, respectively, for $\alpha=e, i$. 
The cold-ion model then reads

$$
\begin{aligned}
& \frac{\partial N_{e}}{\partial t}+\left[\varphi, N_{e}\right]+\nabla_{\|} U_{e}=0, \\
& \frac{\partial A_{\|}}{\partial t}+\left[\varphi, A_{\|}\right]-\nabla_{\|}\left(T_{\|_{e}}-b T_{\perp_{e}}+(1-b) N_{e}-\varphi\right)=0, \\
& \frac{\partial T_{\|_{e}}}{\partial t}+\left[\varphi, T_{\|_{e}}\right]+b\left[T_{\perp_{e}}, T_{\|_{e}}\right]+b\left[N_{e}, T_{\|_{e}}\right] \\
& +b\left[T_{\perp_{e}}, R_{\| \perp_{e}}\right]+b\left[N_{e}, R_{\| \perp_{e}}\right]+\nabla_{\|}\left(Q_{\|_{e}}+2 U_{e}\right)=0, \\
& \frac{\partial T_{\perp_{e}}}{\partial t}+\left[\varphi, T_{\perp_{e}}\right]-2 b\left[T_{\perp_{e}}, N_{e}\right]+2 b\left[T_{\perp_{e}}, R_{\perp \perp e}\right]+2 b\left[N_{e}, R_{\perp \perp e}\right]+\nabla_{\|} Q_{\perp_{e}}=0, \\
& \frac{\partial N_{i}}{\partial t}+\left[\varphi, N_{i}\right]+\nabla_{\|} U_{i}=0, \\
& \frac{\partial}{\partial t}\left(U_{i}+A_{\|}\right)+\left[\varphi, U_{i}\right]+\nabla_{\|} \varphi=0, \\
& \frac{\partial T_{\|_{i}}}{\partial t}+\left[\varphi, T_{\|_{i}}\right]+\nabla_{\|}\left(Q_{\|_{i}}+2 U_{i}\right)=0, \\
& \frac{\partial T_{\perp_{i}}}{\partial t}+\left[\varphi, T_{\perp_{i}}\right]+\nabla_{\|} Q_{\perp_{i}}=0, \\
& \nabla_{\perp}^{2} \varphi=N_{e}-N_{i}, \\
& \nabla_{\perp}^{2} A_{\|}=\frac{b}{1-2 b}\left(U_{e}-U_{i}\right),
\end{aligned}
$$

where we also made use of relation (3.12) to replace $B_{z}$ in favor of $P_{\perp_{e}}$.

If we denote with $D=A_{\|}+U_{i}$ the ion parallel canonical momentum, and consider the energy functional

$$
\begin{aligned}
& H=\frac{1}{2} \int d^{3} x\left((1-b) N_{e}^{2}+\frac{T_{\|_{e}}^{2}}{2}+(1-b) T_{\perp_{e}}^{2}-2 b N_{e} T_{\perp_{e}}-\varphi\left(N_{e}-N_{i}\right)\right. \\
& \left.-A_{\|}\left(U_{e}-U_{i}\right)+A_{\|}^{2}+D^{2}-2 A_{\|} D\right) \\
& =\frac{1}{2} \int d^{3} x\left(N_{e}^{2}+\frac{T_{\|_{e}}^{2}}{2}+T_{\perp_{e}}^{2}-b P_{\perp_{e}}^{2}+|\nabla \varphi|^{2}+\frac{1-2 b}{b}\left|\nabla A_{\|}\right|^{2}+U_{i}^{2}\right),
\end{aligned}
$$

we obtain, from Eqs.(3.18)-(3.27), the following relation

$$
\begin{aligned}
& \dot{H}=\int d^{3} x\left(-T_{\|_{e}} \nabla_{\|} Q_{\|_{e}}+b P_{\perp_{e}} \nabla_{\|} Q_{\perp_{e}}-T_{\perp_{e}} \nabla_{\|} Q_{\perp_{e}}\right. \\
& \left.-(b / 2) T_{\|_{e}}\left[P_{\perp_{e}}, R_{\| \perp_{e}}\right]-2 b T_{\perp_{e}}\left[P_{\perp_{e}}, R_{\perp_{\perp}}\right]\right),
\end{aligned}
$$

where a dot indicates time derivative. It follows in particular that, if the closures on the heat flux and energy-weighted pressure tensor fluctuations are such that the integral on the right-hand side of Eq. (3.29) vanishes, the energy functional $H$ is conserved.

We remark that the functional $H$ consists in the sum of terms involving free energy fluctuations (first three terms on the third line of Eq. (3.28)), energy due to parallel and perpendicular magnetic perturbations (fourth and sixth term, respectively), energy due to electrostatic fluctuations (fifth term) and parallel ion kinetic energy (seventh term).

The expression for the functional $H$ can be constructed starting from the expression 
of the conserved energy functional for the gyrokinetic equations

$\frac{\partial g_{\alpha}}{\partial t}+\frac{c}{B}\left[\delta \phi-\frac{v}{c} \delta A_{\|}+\frac{\mu_{\alpha}}{q_{\alpha}} \delta B_{z}, g_{\alpha}\right]+v \frac{\partial}{\partial z}\left(\widetilde{g}_{\alpha}+\frac{q_{\alpha}}{T_{\alpha}}\left(\delta \phi-\frac{v}{c} \delta A_{\|}+\frac{\mu_{\alpha}}{q_{\alpha}} \delta B_{z}\right) \mathcal{F}_{e q_{\alpha}}\right)=0$

where $\widetilde{g}_{\alpha}=\widetilde{f}_{\alpha}+\left(q_{\alpha} / T_{\alpha}\right)(v / c) \mathcal{F}_{e q_{\alpha}} A_{\|}$, for $\alpha=e, i$ and where Eqs. (3.10)-(3.12) have to be used to determine the electromagnetic perturbations $\delta \phi, \delta A_{\|}$and $\delta B_{z}$.

Equation (3.30) is obtained from the original gyrokinetic equation (2.1) by assuming $\widetilde{f}_{\alpha} / \mathcal{F}_{e q_{\alpha}}=\mathcal{O}(\epsilon)$, applying the ordering (2.14) and retaining only terms of order $\epsilon^{2}$. The gyroaveraging operators have also been approximated by $J_{0} \sim 1$ and $J_{1} \sim k_{\perp} \rho_{\perp_{\alpha}} / 2$, which can be seen a posteriori to be consistent with the asymptotic limits $\tau \ll 1$ and $\delta \ll 1$ (the model (3.18)-(3.27) could indeed be alternatively derived directly by taking moments of Eqs. (3.30) and then applying the limit $\tau \ll 1, \delta \ll 1$ to the resulting equations). The system composed by Eqs. (3.30), (3.10), (3.11) and (3.12) admits the conserved energy functional

$$
\mathcal{H}=\frac{1}{2} \sum_{\alpha=e, i} \int d^{3} x d \mathcal{W}_{\alpha}\left(T_{\alpha} \frac{\widetilde{g}_{\alpha}^{2}}{\mathcal{F}_{e q_{\alpha}}}+q_{\alpha}\left(\delta \phi-\frac{v}{c} \delta A_{\|}+\frac{\mu_{\alpha}}{q_{\alpha}} \delta B_{z}\right) \widetilde{g}_{\alpha}\right)
$$

One can then suppose that the perturbed functions $\widetilde{g}_{\alpha}$ are amenable to the following expansion in terms of Hermite-Laguerre polynomials, truncated to account for the dynamical variables present in the fluid model, that is, density, canonical momentum, parallel and perpendicular temperatures:

$$
\widetilde{g}_{\alpha}=\mathcal{F}_{e q_{\alpha}}\left[\frac{\widetilde{N}_{\alpha}}{n_{0}}+\left(\frac{\widetilde{U}_{\alpha}}{v_{t \alpha}}+\frac{q_{\alpha} A_{\|}}{m_{\alpha} v_{t \alpha} c}\right) \frac{v}{v_{t \alpha}}+\frac{1}{2} \frac{\widetilde{T}_{\|_{\alpha}}}{T_{\alpha}}\left(\frac{v^{2}}{v_{t \alpha}^{2}}-1\right)-\frac{\widetilde{T}_{\perp_{\alpha}}}{T_{\alpha}}\left(1-\frac{\mu_{\alpha} B}{T_{\alpha}}\right)\right]
$$

with $v_{t \alpha}=\sqrt{T_{\alpha} / m_{\alpha}}$. One can insert expression (3.32) into the functional (3.31) and then take the limit $\tau \ll 1$ and $\delta \ll 1$. The resulting functional is namely (3.28). Therefore the origin of the expression for the functional (3.28) is explained by the consistency with the conserved energy functional of the underlying gyrokinetic model. A similar procedure was used to establish energy conservation theorems for gyrofluid models in Scott (2010).

With regard to the second model, valid for the sub-ionic scales, its equations can be 
rewritten as

$$
\begin{aligned}
& \frac{\partial N_{e}}{\partial t}+\left[\varphi, N_{e}\right]+\frac{\tau}{1+\tau b}\left[N_{e}, T_{\perp_{e}}\right]+\nabla_{\|} U_{e}=0, \\
& \frac{\partial A_{\|}}{\partial t}-\nabla_{\|}\left(T_{\|_{e}}-\frac{b}{1+\tau b} T_{\perp_{e}}+\frac{1-b}{1+\tau b} N_{e}-\varphi\right)=0, \\
& \frac{\partial T_{\|_{e}}}{\partial t}+\left[\varphi, T_{\|_{e}}\right]+\frac{b}{1+\tau b}\left[T_{\perp_{e}}, T_{\|_{e}}\right]+b \frac{1+\tau}{1+\tau b}\left[N_{e}, T_{\|_{e}}\right] \\
& +\frac{b}{1+\tau b}\left[T_{\perp_{e}}, R_{\| \perp_{e}}\right]+b \frac{1+\tau}{1+\tau b}\left[N_{e}, R_{\| \perp_{e}}\right]+\nabla_{\|}\left(Q_{\|_{e}}+2 U_{e}\right)=0, \\
& \frac{\partial T_{\perp_{e}}}{\partial t}\left[\varphi, T_{\perp_{e}}\right]-\frac{3 \tau b+2 b}{1+\tau b}\left[T_{\perp_{e}}, N_{e}\right] \\
& +\frac{2 b}{1+\tau b}\left[T_{\perp_{e}}, R_{\perp \perp e}\right]+\frac{2 b(1+\tau)}{1+\tau b}\left[N_{e}, R_{\perp \perp e}\right]+\nabla_{\|} Q_{\perp_{e}}=0, \\
& \varphi=\tau \frac{b-1}{1+\tau b} N_{e}+\frac{\tau b}{1+\tau b} T_{\perp_{e}}, \\
& \nabla_{\perp}^{2} A_{\|}=\frac{b}{1-2 b} U_{e},
\end{aligned}
$$

where we made use of Eq. (3.17) and of the relation $P_{\perp_{e}}=T_{\perp_{e}}+N_{e}$ to express $B_{z}$ in terms of $N_{e}$ and $T_{\perp_{e}}$.

By means of a procedure analogous to that adopted for the cold-ion model, one obtains that the energy functional for the system (3.33)-(3.38) reads

$$
H=\frac{1}{2} \int d^{3} x\left(N_{e}^{2}+\frac{1-2 b}{2 b}\left|\nabla A_{\|}\right|^{2}+\frac{T_{\|_{e}}^{2}}{2}+T_{\perp_{e}}^{2}-\varphi N_{e}-\frac{\tau b}{1+\tau b} N_{e} P_{\perp_{e}}-\frac{b}{1+\tau b} P_{\perp_{e}}^{2}\right)
$$

and that its time evolution is governed by

$$
\begin{aligned}
& \dot{H}=\int d^{3} x\left(T_{\|_{e}}\left(\frac{1}{2}\left[B_{z}, R_{\| \perp_{e}}\right]-\nabla_{\|} Q_{\|_{e}}\right)\right. \\
& \left.+\left(T_{\perp_{e}}-\frac{\tau b}{1+\tau b} N_{e}-\frac{b}{1+\tau b} P_{\perp_{e}}\right)\left(2\left[B_{z}, R_{\perp \perp_{e}}\right]-\nabla_{\|} Q_{\perp_{e}}\right)\right) .
\end{aligned}
$$

Also in this case, depending on the closure relation, the functional $H$ can be conserved.

A natural question that arises in connection with the energy conservation properties of the systems, concerns the existence of a Hamiltonian structure for such models. Typically, in the absence of dissipative terms, fluid models for plasmas expressed in terms of Eulerian variables, possess a noncanonical Hamiltonian structure (see, e.g. Morrison (1998)). In particular, reduced drift or gyrofluids models in their two-dimensional limit possess noncanonical Poisson brackets of Lie-Poisson type (see, e.g. Thiffeault \& Morrison (2000); Waelbroeck \& Tassi (2012); Hazeltine et al. (1987)) and the extension of the Hamiltonian structure to three dimensions, in the limit $k_{z} / k_{\perp} \ll 1$, can be obtained following the prescription of Tassi et al. (2010). Clearly, the possibility for the existence of a Hamiltonian structure depends on the adopted closures. A systematic identification of all possible closures leading to Hamiltonian models with a Lie-Poisson bracket lies outside the scope of this paper, but we note that, for example, by choosing the closure

$$
\begin{array}{r}
Q_{\|_{e}}=Q_{\perp_{e}}=Q_{\|_{i}}=Q_{\perp_{i}}=0, \\
R_{\perp \perp e}=0, \quad R_{\| \perp_{e}}=-\frac{1}{1+2 b} T_{\|_{e}} .
\end{array}
$$


the cold-ion model acquires a Hamiltonian structure consisting of the functional (3.28) as Hamiltonian with the Poisson bracket

$$
\{F, G\}=\{F, G\}_{e}-\{F, G\}_{i}
$$

where

$$
\begin{aligned}
& \{F, G\}_{e}=\int d^{3} x\left(N_{e}\left(\left[F_{N_{e}}, G_{N_{e}}\right]+2 \frac{1+b}{1+2 b}\left[F_{T_{\|_{e}}}, G_{T_{\|_{e}}}\right]\right)\right. \\
& +A_{\|}\left(\left[F_{N_{e}}, G_{A_{\|}}\right]+\left[F_{A_{\|}}, G_{N_{e}}\right]+2\left(\left[F_{A_{\|}}, G_{T_{\|_{e}}}\right]+\left[F_{T_{\|_{e}}}, G_{A_{\|}}\right]\right)\right)+ \\
& T_{\|_{e}}\left(\left[F_{N_{e}}, G_{T_{\|_{e}}}\right]+\left[F_{T_{\|_{e}}}, G_{N_{e}}\right]+\frac{1+3 b}{1+2 b}\left[F_{T_{\|_{e}}}, G_{T_{\|_{e}}}\right]\right) \\
& +T_{\perp_{e}}\left(\left[F_{N_{e}}, G_{T_{\perp_{e}}}\right]+\left[F_{T_{\perp_{e}}}, G_{N_{e}}\right]-\frac{2 b}{1+2 b}\left[F_{T_{\|_{e}}}, G_{T_{\|_{e}}}\right]+\frac{1+b}{b}\left[F_{T_{\perp_{e}}}, G_{T_{\perp_{e}}}\right]\right) \\
& \left.+F_{N_{e}} \frac{\partial}{\partial z} G_{A_{\|}}+F_{A_{\|}} \frac{\partial}{\partial z} G_{N_{e}}+2 F_{A_{\|}} \frac{\partial}{\partial z} G_{T_{\|_{e}}}+2 F_{T_{\|_{e}}} \frac{\partial}{\partial z} G_{A_{\|}}\right),
\end{aligned}
$$

and

$$
\begin{aligned}
& \{F, G\}_{i}=\int d^{3} x\left(N_{i}\left[F_{N_{i}}, G_{N_{i}}\right]\right. \\
& +D\left(\left[F_{N_{i}}, G_{D}\right]+\left[F_{D}, G_{N_{i}}\right]+2\left(\left[F_{D}, G_{T_{\|_{i}}}\right]+\left[F_{T_{\|_{i}}}, G_{D}\right]\right)\right) \\
& +T_{\|_{i}}\left(\left[F_{N_{i}}, G_{T_{\|_{i}}}\right]+\left[F_{T_{\|_{i}}}, G_{N_{i}}\right]+2\left[F_{T_{\|_{i}}}, G_{T_{\|_{i}}}\right]\right) \\
& +T_{\perp_{i}}\left(\left[F_{N_{i}}, G_{T_{\perp_{i}}}\right]+\left[F_{T_{\perp_{i}}}, G_{N_{i}}\right]\right) \\
& \left.+F_{N_{i}} \frac{\partial}{\partial z} G_{D}+F_{D} \frac{\partial}{\partial z} G_{N_{i}}+2 F_{T_{\|_{i}}} \frac{\partial}{\partial z} G_{D}+2 F_{D} \frac{\partial}{\partial z} G_{T_{\|_{i}}}\right) .
\end{aligned}
$$

Here, the subscripts on functionals indicate functional derivatives. The bracket (3.43) is the direct sum of two Poisson brackets, acting on electron and ion moments, respectively. The coupling in the dynamics, on the other hand, comes from the Hamiltonian (3.28). It can be verified that the two operations (3.44) and (3.45) satisfy bilinearity, antisymmetry as well as Leibniz and Jacobi identities.

\subsection{Particle moment formulation}

The cold-ion model (3.2)-(3.12) and the sub-ion scale model consisting of Eqs. (3.2)(3.5), together with Eqs. (3.15) and (3.17), are written in terms of gyrofluid moments. For a more complete physical interpretation, it is however important to determine their expression also in terms of particle moments. This is accomplished in the following Secs. 3.4.1 and 3.4.2.

\subsubsection{Cold-ion limit}

In the limit of cold ions and for scales larger than the electron thermal gyroradius, the transformation between the gyrofluid and the particle moments is given by Brizard 
(1992) as

$$
\begin{aligned}
N_{e} & =n_{e}-\widehat{B_{z}}, & U_{e} & =u_{e}, \\
P_{\|_{e}} & =p_{\|_{e}}-\widehat{B_{z}}, & P_{\perp_{e}} & =p_{\perp_{e}}-2 \widehat{B_{z}}, \\
N_{i} & =n_{i}-\widehat{B_{z}}-\nabla_{\perp}^{2} \widehat{\varphi}, & U_{i} & =u_{i}, \\
P_{\|_{i}} & =p_{\|_{i}}-\widehat{B_{z}}-\nabla_{\perp}^{2} \widehat{\varphi}, & P_{\perp_{i}} & =p_{\perp_{i}}-2 \widehat{B_{z}}-2 \nabla_{\perp}^{2} \widehat{\varphi}, \\
Q_{\|_{\alpha}} & =q_{\|_{\alpha}}, & Q_{\perp_{\alpha}} & =q_{\perp_{\alpha}}, \\
R_{x_{\alpha}} & =r_{x_{\alpha}}-2 \widehat{B_{z}}, & R_{\perp_{\alpha}}^{(\perp)} & =r_{\perp_{\alpha}}^{(\perp)}-6 \widehat{B_{z}},
\end{aligned}
$$

for $\alpha=e, i$. In Eqs. (3.46)-(3.51), the quantities $n_{\alpha}, u_{\alpha}, p_{\|_{\alpha}}, p_{\perp_{\alpha}}, q_{\|_{\alpha}}, q_{\perp_{\alpha}}, r_{x_{\alpha}}$ and $r_{\perp_{\alpha}}^{(\perp)}$, for $\alpha=e, i$, indicate the moments with respect to the perturbed particle distribution function. We indicated with a hat the electromagnetic fluctuations expressed in terms of the particle moments, so that, for instance, $B_{z}\left(P_{\perp_{e}}\right)=\widehat{B_{z}}\left(p_{\perp_{e}}\right)$. Transformations (3.46)(3.51) are consistent with the asymptotic limits and the assumptions made to derive Eqs. (3.2)-(3.12).

In terms of the particle moments, the system (3.2)-(3.12) can be written (after suppression of the hats) as

$$
\begin{aligned}
& \frac{d}{d t}\left(n_{e}-B_{z}\right)+\nabla_{\|} u_{e}=0, \\
& \frac{d A_{\|}}{d t}-\nabla_{\|} p_{\|_{e}}+\frac{\partial \varphi}{\partial z}=0, \\
& \frac{d}{d t}\left(p_{\|_{e}}-B_{z}\right)-\left[B_{z}, p_{\|_{e}}\right]+\left[B_{z}, n_{e}\right]-\left[B_{z}, r_{\| \perp e}\right]+\nabla_{\|}\left(q_{\|_{e}}+3 u_{e}\right)=0, \\
& \frac{d}{d t}\left(p_{\perp_{e}}-2 B_{z}\right)+2\left[B_{z}, n_{e}\right]-2\left[B_{z}, r_{\perp \perp e}\right]+\nabla_{\|}\left(u_{e}+q_{\perp_{e}}\right)=0, \\
& \frac{d}{d t}\left(n_{i}-B_{z}-\nabla_{\perp}^{2} \varphi\right)+\nabla_{\|} u_{i}=0 \\
& \frac{d}{d t}\left(u_{i}+A_{\|}\right)+\frac{\partial \varphi}{\partial z}=0 \\
& \frac{d}{d t}\left(p_{\|_{i}}-B_{z}-\nabla_{\perp}^{2} \varphi\right)+\nabla_{\|}\left(q_{\|_{i}}+3 u_{i}\right)=0, \\
& \frac{d}{d t}\left(p_{\perp_{i}}-2 B_{z}-2 \nabla_{\perp}^{2} \varphi\right)+\nabla_{\|}\left(u_{i}+q_{\perp_{i}}\right)=0, \\
& n_{e}=n_{i}, \\
& \nabla_{\perp}^{2} A_{\|}=\frac{\beta_{e}}{2}\left(u_{e}-u_{i}\right) \\
& B_{z}=-\frac{\beta_{e}}{2} p_{\perp_{e}}
\end{aligned}
$$

where we introduced the operator

$$
\frac{d}{d t}=\frac{\partial}{\partial t}+[\varphi,]
$$

We remark that, in terms of the particle perturbed moments, Eqs. (3.60) and (3.62) take the familiar form of the quasi-neutrality condition and of the balance between magnetic and pressure perturbations (in this case, the latter are restricted to the electron pressure because of the cold ion limit). In particular, from Eqs. (3.60), (3.61), (3.52) and (3.56), 
one gets

$$
\frac{d \nabla_{\perp}^{2} \varphi}{d t}+\frac{2}{\beta_{e}} \nabla_{\|} \nabla_{\perp}^{2} A_{\|}=0
$$

which corresponds to the usual vorticity equation of reduced magnetohydrodynamics.

By imposing isotropic and isothermal electrons, i.e. $p_{\|_{e}}=p_{\perp_{e}}=n_{e}=-\left(2 / \beta_{e}\right) B_{z}$, one obtains, from Eqs. (3.52), (3.53) and (3.57), the set of equations

$$
\begin{aligned}
\frac{d B_{z}}{d t}-\frac{\beta_{e}}{2+\beta_{e}} \nabla_{\|}\left(\frac{2}{\beta_{e}} \nabla_{\perp}^{2} A_{\|}+u_{i}\right) & =0, \\
\frac{d A_{\|}}{d t}+\frac{2}{\beta_{e}} \nabla_{\|} B_{z}+\frac{\partial \varphi}{\partial z} & =0, \\
\frac{d u_{i}}{d t}-\frac{2}{\beta_{e}} \nabla_{\|} B_{z} & =0,
\end{aligned}
$$

which, together with the vorticity equation (3.64), forms the closed system of Hall reduced MHD (Schekochihin et al. 2009). From such system, if the evolution equation (3.67) is replaced by $u_{i}=0$, one recovers the three-field low-ion beta model of Boldyrev et al. (2013). On the other hand, Eqs. (3.52) and (3.53) are compatible with Eqs. (107) and (106) of Boldyrev et al. (2013), and the model (3.52)-(3.62) generalizes the electron dynamics to account for anisotropic pressure evolution, when compared to the models of Boldyrev et al. (2013). However, the ion dynamic is treated in a different way, for in our case the cold ion, low ion-beta limit is taken.

\subsubsection{Sub-ion limit}

In this regime, for the electron moments, the mapping between gyrofluid and particle moments is the same as in Sec. 3.4.1 and is thus given by

$$
\begin{aligned}
N_{e} & =n_{e}-\widehat{B_{z}}, & U_{e} & =u_{e}, \\
P_{\|_{e}} & =p_{\|_{e}}-\widehat{B_{z}}, & P_{\perp_{e}} & =p_{\perp_{e}}-2 \widehat{B_{z}}, \\
Q_{\|_{e}} & =q_{\|_{e}}, & Q_{\perp_{e}} & =q_{\perp_{e}}, \\
R_{x_{e}} & =r_{x_{e}}-2 \widehat{B_{z}}, & R_{\perp_{e}}^{(\perp)} & =r_{\perp_{e}}^{(\perp)}-6 \widehat{B_{z}},
\end{aligned}
$$

When transformed in terms of particle moments, the sub-ion model becomes

$$
\begin{aligned}
& \frac{d}{d t}\left(n_{e}-B_{z}\right)-\left[B_{z}, p_{\perp_{e}}\right]+\nabla_{\|} u_{e}=0 \\
& \frac{d A_{\|}}{d t}-\nabla_{\|} p_{\|_{e}}+\frac{\partial \varphi}{\partial z}=0 \\
& \frac{d}{d t}\left(p_{\|_{e}}-B_{z}\right)-\left[B_{z}, p_{\|_{e}}\right]-\left[B_{z}, p_{\perp_{e}}\right]+\left[B_{z}, n_{e}\right]-\left[B_{z}, r_{\| \perp e}\right]+\nabla_{\|}\left(q_{\|_{e}}+3 u_{e}\right)=0 \\
& \frac{d}{d t}\left(p_{\perp_{e}}-2 B_{z}\right)-4\left[B_{z}, p_{\perp_{e}}\right]+2\left[B_{z}, n_{e}\right]-2\left[B_{z}, r_{\perp \perp e}\right]+\nabla_{\|}\left(u_{e}+q_{\perp_{e}}\right)=0 \\
& \varphi=-\tau n_{e} \\
& \nabla_{\perp}^{2} A_{\|}=\frac{\beta_{e}}{2} u_{e}, \\
& B_{z}=-\tau \frac{\beta_{e}}{2} n_{e}-\frac{\beta_{e}}{2} p_{\perp_{e}} .
\end{aligned}
$$


Note that the electromagnetic fluctuations $\varphi, A_{\|}$and $B_{z}$ are entirely expressed in terms of electron particle moments and agree with those of Schekochihin et al. (2009) and Boldyrev et al. (2013). The evolution equations for the electron moments, on the other hand, remain unchanged with respect to those of the previous section because in both cases we assumed $\delta^{2} \Delta_{s} \ll 1$. Furthermore, if instead of Eqs. (3.74) and (3.75), an isothermal isotropic electron pressure $\left(p_{\|_{e}}=p_{\perp_{e}}=n_{e}\right)$ is prescribed, the system reduces to

$$
\begin{aligned}
& \left(1+(1+\tau) \frac{\beta_{e}}{2}\right) \frac{\partial n_{e}}{\partial t}+\frac{2}{\beta_{e}} \nabla_{\|} \nabla_{\perp}^{2} A_{\|}=0, \\
& \frac{\partial A_{\|}}{\partial t}-(1+\tau) \nabla_{\|} n_{e}=0,
\end{aligned}
$$

which corresponds, although written with a different normalization, to the model of Boldyrev et al. (2013) for the sub-proton scales.

We remark that, for the ions, the transformation from gyrofluid to particle moments, in the limit $k_{\perp} \rho_{i} \gg 1$ is not invertible and yields, to leading order,

$$
\begin{aligned}
n_{i} & =p_{\|_{i}}=p_{\perp_{i}}=-\frac{\varphi}{\tau}, \\
u_{i} & =0
\end{aligned}
$$

which corresponds to an isothermal isotropic fluid with a Boltzmann response to the potential fluctuations and with negligible parallel ion velocity. This corresponds to the hypotheses adopted in Boldyrev et al. (2013).

\section{A reduced FLR-Landau fluid model}

The starting point is provided by the FLR-Landau fluid model discussed in Section 5 of Sulem \& Passot (2015), considered at the lowest relevant order relatively to the ordering defined in Eq. (2.14), and written in a non-dimensional form, using the units defined by Eqs. (2.12). In contrast with the previous section, it is not convenient here to separate everywhere the equilibrium profiles from the fluctuations. We thus keep the original notations of the various variables and indicate, when needed, fluctuations by means of the symbol $\delta$. Furthermore, we use the notation $u_{\| \alpha}$ for the parallel (hydrodynamic) velocity of particles of species $\alpha$, which was referred to as $u_{\alpha}$ in the previous sections.

When neglecting electron inertia, the electron velocity equation leads to

$$
\mathbf{u}_{\perp e}=\mathbf{E} \times \widehat{\mathbf{b}}-\frac{1}{n} \widehat{\mathbf{b}} \times \nabla \cdot \mathbf{p}_{e}
$$

where $\widehat{\mathbf{b}}$ denotes the unit vector in the direction of the ambient field, and $n$ is the density of both the ions and the electrons, due to electric quasi-neutrality.

Within the ordering defined by Eqs. (2.14), and assuming isotropic equilibrium pressures and scales large compared with the electron gyroradius (which makes electron finite Larmor radius effect negligible) one has

$$
\nabla \cdot \mathbf{p}_{e}=\nabla p_{\perp e}+\nabla_{\|}\left(p_{\| e}-p_{\perp e}\right) \widehat{\mathbf{b}}
$$

It follows that, to leading order, $\left(\nabla \cdot \mathbf{p}_{e}\right)_{\perp}=\nabla_{\perp} p_{\perp e}$ and $\widehat{\mathbf{b}} \cdot\left(\nabla \cdot \mathbf{p}_{e}\right)=\nabla_{\|} p_{\| e}$, where, in the present asymptotics, transverse differential operators are defined with respect to the guide field. Similarly, one can approximate $\widehat{\mathbf{b}} \times \nabla$ by $\widehat{\mathbf{z}} \times \nabla$, which gives

$$
\mathbf{u}_{\perp e}=\widehat{\mathbf{z}} \times \nabla\left(\varphi-p_{\perp e}\right) .
$$


From Faraday equation and Ohm's law $\left(\mathbf{E}=-\mathbf{u}_{e} \times \mathbf{B}-(1 / n) \nabla \cdot \mathbf{p}_{e}\right)$, one has to leading order

$$
\frac{d B_{z}}{d t}-\left[p_{\perp e}, B_{z}\right]-\nabla_{\|} u_{\| e}+\nabla \cdot \mathbf{u}_{e}-\left[p_{\perp e}, n\right]=0,
$$

where, when not differently specified, the parallel component of the various vector fields and their $z$-components can be identified. In order to eliminate $\nabla \cdot \mathbf{u}_{e}$, it is convenient to use the electron continuity equation

$$
\frac{d n}{d t}-\left[p_{\perp e}, n\right]+\nabla \cdot \mathbf{u}_{e}=0
$$

One gets

$$
\frac{d}{d t}\left(n-B_{z}\right)+\nabla_{\|} u_{\| e}-\left[B_{z}, p_{\perp e}\right]=0 .
$$

The equation for the electron pressures read

$$
\begin{aligned}
& \partial_{t} p_{\| e}+\mathbf{u}_{e} \cdot \nabla p_{\| e}+p_{\| e} \nabla \cdot \mathbf{u}_{e}+2 p_{\| e} \widehat{\mathbf{b}} \cdot \nabla \mathbf{u}_{e} \cdot \widehat{\mathbf{b}}+\left(\nabla \cdot \mathbf{q}_{e}\right): \boldsymbol{\tau}=0 \\
& \partial_{t} p_{\perp e}+\mathbf{u}_{e} \cdot \nabla p_{\perp e}+2 p_{\perp e} \nabla \cdot \mathbf{u}_{e}-p_{\perp e} \widehat{\mathbf{b}} \cdot \nabla \mathbf{u}_{e} \cdot \widehat{\mathbf{b}}+\frac{1}{2}\left(\nabla \cdot \mathbf{q}_{e}\right): \mathbf{n}=0
\end{aligned}
$$

where $\boldsymbol{\tau}=\widehat{\mathbf{b}} \otimes \widehat{\mathbf{b}}$ and $\mathbf{n}=\mathbf{I}-\boldsymbol{\tau}$. To leading order, one can write

$$
\begin{aligned}
& \left(\nabla \cdot \mathbf{q}_{e}\right): \boldsymbol{\tau}=\nabla_{\|} q_{\| e}+\nabla \cdot \mathbf{S}_{\perp e}^{\|} \\
& \frac{1}{2}\left(\nabla \cdot \mathbf{q}_{e}\right): \mathbf{n}=\nabla_{\|} q_{\perp e}+\nabla \cdot \mathbf{S}_{\perp r}^{\perp} .
\end{aligned}
$$

Here, the nongyrotropic heat fluxes contributions, described by the vectors $\mathbf{S}_{\perp e}^{\|}$and $\mathbf{S}_{\perp r}^{\perp}$, are estimated for scales much larger than the electron gyroscales by Eqs. (3.8) and (3.9) of Sulem \& Passot (2015), in the form

$$
\begin{aligned}
& \mathbf{S}_{\perp e}^{\|}=-\frac{1}{B} \widehat{\mathbf{b}} \times\left(p_{\perp e} \nabla T_{\| e}+\nabla \widetilde{r}_{\| \perp e}\right) \\
& \mathbf{S}_{\perp e}^{\perp}=-\frac{2}{B} \widehat{\mathbf{b}} \times\left(p_{\perp_{e}} \nabla T_{\perp e}+\nabla \widetilde{r}_{\perp \perp e}\right),
\end{aligned}
$$

where, at the order of the present analysis, the fluctuations of the magnetic field originating from the local Larmor radius, are to be retained. Furthermore, the contribution of the gyrotropic fourth-order cumulant $\widetilde{r}_{\| \perp e}$, not retained in Eq. (3.7) of Sulem \& Passot (2015), turns out to be potentially relevant, for example within the closure model defined by Eqs. (3.24)-(3.27) and (3.17)-(3.19) of the above reference, where $\widetilde{r}_{\| \perp e}$ scales like $M / m$ in the units of that paper. Differently, within this framework, $\widetilde{r}_{\perp \perp e}$ is negligible.

Combining with the equation for $B_{z}$, one gets

$$
\begin{aligned}
& \frac{d}{d t}\left(p_{\| e}-B_{z}\right)-\left[B_{z}, p_{\perp e}\right]-\left[B_{z}, p_{\| e}-n+\widetilde{r}_{\| \perp e}\right]+\nabla_{\|}\left(3 u_{\| e}+q_{\| e}\right)=0 \\
& \frac{d}{d t}\left(p_{\perp e}-2 B_{z}\right)-4\left[B_{z}, p_{\perp e}\right]+2\left[B_{z}, n-\widetilde{r}_{\perp \perp e}\right]+\nabla_{\|}\left(u_{\| e}+q_{\perp e}\right)=0 .
\end{aligned}
$$

Ampère law directly gives that, to leading order,

$$
u_{\| e}=u_{\| i}+\frac{2}{\beta_{e}} \nabla_{\perp}^{2} A_{\|} .
$$

Equating the expressions of the electric field given by Ohm's law and Faraday equation 
(taken in the form $\mathbf{E}=-\nabla \varphi-\partial_{t} \mathbf{A}$ ), leads for the parallel component to

$$
\frac{d A_{\|}}{d t}-\nabla_{\|} p_{\| e}+\partial_{z} \varphi=0
$$

and for the perpendicular one to

$$
-\nabla_{\perp} \varphi=\widehat{\mathbf{z}} \times\left(\mathbf{u}_{i}-\frac{2}{\beta_{e}} \nabla \times \mathbf{B}\right)-\nabla_{\perp} p_{\perp e},
$$

or when writing $\mathbf{u}_{\perp i}=-\nabla_{\perp} \chi_{c}+\nabla_{\perp} \times\left(\chi_{s} \widehat{\mathbf{z}}\right)$ and taking the transverse divergence,

$$
\nabla_{\perp}^{2}\left(\varphi+\chi_{s}-\frac{2}{\beta_{e}} B_{z}-p_{\perp e}\right)=0 .
$$

Note that the quantities $\varphi, B_{z}$ and $A_{\|}$determine the full electromagnetic field, as the condition $\mathbf{B}=\nabla \times \mathbf{A}$, together with the gauge condition $\nabla \cdot \mathbf{A}=0$ gives $\nabla_{\perp}^{2} \mathbf{A}_{\perp}=$ $\widehat{\mathbf{z}} \times \nabla B_{z}-\partial_{z} \nabla A_{\|}$

One now turns to the equation for the ion velocity where the electric field has been expressed using Ohm's law. It reads

$$
\frac{D^{(i)}}{D t} \mathbf{u}_{i}+\frac{1}{n} \nabla \cdot\left(\mathbf{p}^{(G)}+\mathbf{\Pi}\right)-\frac{2}{\beta_{e}} \frac{1}{n}(\nabla \times \mathbf{B}) \times \mathbf{B}=0,
$$

where $D^{(i)} / D t=\partial_{t}+\mathbf{u}_{i} \cdot \nabla$, while $\mathbf{p}^{(G)}$ denotes the total gyrotropic pressure tensor and $\boldsymbol{\Pi}$ the non-gyrotropic contribution to the ion pressure. One writes $\nabla \cdot \boldsymbol{\Pi}=\nabla \cdot(-\mathcal{A} \mathbf{n}+$ $\left.\mathcal{B} \boldsymbol{\epsilon} \cdot \mathbf{b}+\mathbf{b} \otimes \boldsymbol{\Pi}_{\|}+\boldsymbol{\Pi}_{\|} \otimes \mathbf{b}\right)$, where $\mathbf{n}=\mathbf{I}-\mathbf{b} \otimes \mathbf{b}$. The scalar quantities $\mathcal{A}$ and $\mathcal{B}$, together with the vector $\boldsymbol{\Pi}_{\|}$, are defined by Eqs. (5.7)-(5.9) of Sulem \& Passot (2015).

The equation for $u_{\| i}$ is obtained by estimating $\boldsymbol{\Pi}_{\|}$from Eq. (5.9) of Sulem \& Passot (2015), which, to leading order, gives

$$
\partial_{t} u_{\| i}+\left[u_{\| i}, \chi_{s}\right]+\nabla_{\|}\left(\tau p_{\| i}+p_{\| e}\right)+\tau \widehat{\mathfrak{C}}_{2}\left(\nabla_{\perp}^{2} \nabla_{\|} p_{\| e}+\nabla_{\|}(\mathbf{n}: \nabla \mathbf{E})\right)=0,
$$

where it is sufficient to write $(\mathbf{n}: \nabla \mathbf{E})=-\nabla_{\perp}^{2} \varphi$. Furthermore, $\widehat{\mathfrak{C}}_{2}$ is an operator which in Fourier space reduces to the multiplication by the factor

$$
\mathfrak{C}_{2}=\frac{1}{b_{i}}\left(1-\Gamma_{0}\left(b_{i}\right)\right) .
$$

Here, $\Gamma_{n}\left(b_{i}\right)=\exp \left(-b_{i}\right) I_{n}\left(b_{i}\right)$ where $I_{n}$ denotes the modified Bessel function of order $n$. The variable $b_{i}$, which is defined in the physical variables by $b_{i}=\left(k_{\perp}^{2} \rho_{i}^{2}\right) / 2$ (where $\rho_{i}$ holds for the ion thermal gyroradius) reduces in the present units to $b_{i}=\tau k_{\perp}^{2}$. Furthermore, in the limit $b_{i} \rightarrow 0, \mathfrak{C}_{2} \rightarrow 1$ while for $b \rightarrow \infty, \mathfrak{C}_{2}=1 / b_{i}+\mathcal{O}\left(1 / b_{i}^{3 / 2}\right)$.

Considering the transverse component of Eq. (4.19) and taking the transverse divergence, one gets to leading order the transverse pressure balance equation

$$
\nabla_{\perp}^{2}\left(\tau p_{\perp i}+p_{\perp e}+\frac{2}{\beta_{e}} B_{z}-\mathcal{A}\right)=0,
$$

where, from Eq. (5.7) of Sulem \& Passot (2015), $\mathcal{A}=\tau\left(\widehat{\mathfrak{A}}_{1} \omega_{\| i}+\widehat{\mathfrak{A}}_{2} T_{\perp i}\right)$, with $\omega_{\| i}=$ $-\nabla_{\perp}^{2} \chi_{s}$ denoting the parallel ion vorticity. The operators $\widehat{\mathfrak{A}}_{1}$ and $\widehat{\mathfrak{A}}_{2}$ reduce in Fourier space to the multiplicative factors

$$
\begin{aligned}
& \mathfrak{A}_{1}=1-\frac{\Gamma_{1}\left(b_{i}\right)}{b_{i}\left[\Gamma_{0}\left(b_{i}\right)-\Gamma_{1}\left(b_{i}\right)\right]}+\frac{\Gamma_{1}\left(b_{i}\right)}{\Gamma_{0}\left(b_{i}\right)} \\
& \mathfrak{A}_{2}=-\frac{\Gamma_{1}\left(b_{i}\right)}{\Gamma_{0}\left(b_{i}\right)}
\end{aligned}
$$


For $b_{i} \rightarrow 0, \mathfrak{A}_{1} \rightarrow 1 / 2$ and $\mathfrak{A}_{2} \rightarrow 0$, while for $b_{i} \rightarrow \infty, \mathfrak{A}_{1} \sim 1 / b_{i}$ and $\mathfrak{A}_{2} \rightarrow-1$.

Equation (4.22) rewrites

$$
\nabla_{\perp}^{2}\left[\frac{2}{\beta_{e}} B_{z}+\tau n+p_{\perp e}-\tau \widehat{\mathfrak{A}}_{1} \omega_{\| i}+\tau\left(1-\widehat{\mathfrak{A}}_{2}\right) T_{\perp i}\right]=0 .
$$

Combining this equation with Eq. (4.18) also gives

$$
\nabla_{\perp}^{2}\left[\tau p_{\perp i}+\varphi+\chi_{s}-\tau \widehat{\mathfrak{A}}_{1} \omega_{\| i}-\tau \widehat{\mathfrak{A}}_{2} T_{\perp i}\right]=0 .
$$

The equation for the parallel ion vorticity reads

$$
\frac{D^{(i)} \omega_{\| i}}{D t}-(\omega \cdot \nabla) u_{\| i}+\left(\nabla \cdot \mathbf{u}_{i}\right) \omega_{\| i}+\widehat{\mathbf{z}} \cdot \nabla \times\left[\frac{1}{n} \nabla \cdot\left(\mathbf{p}_{i}+\mathbf{p}_{e}\right)\right]-\frac{2}{\beta_{e}} \widehat{\mathbf{z}} \cdot \nabla \times\left[\frac{1}{n}(\nabla \times \mathbf{B}) \times \mathbf{B}\right]=0 .
$$

The ion continuity equation shows that $\nabla \cdot \mathbf{u}_{i}$ is second-order in magnitude, making $\left(\nabla \cdot \mathbf{u}_{i}\right) \omega_{\| i}$ negligible and indicating that, to leading order, $\mathbf{u}_{\perp i}=-\widehat{\mathbf{z}} \times \nabla_{\perp} \chi_{s}$, and thus $D^{(i)} / d t=\partial_{t}+\left[\cdot, \chi_{s}\right]$. The ion vorticity equation rewrites

$$
\partial_{t} \omega_{\| i}+\left[\omega_{\| i}, \chi_{s}\right]-\left[n, \tau p_{\perp i}+p_{\perp e}+\frac{2}{\beta_{e}} B_{z}\right]+\frac{2}{\beta_{e}} \nabla_{\|} \nabla_{\perp}^{2} A_{\|}-\nabla_{\perp}^{2} \mathcal{B}=0,
$$

where, from Eq. (5.8) of Sulem \& Passot (2015),

$$
\mathcal{B}=\tau\left[\widehat{\mathfrak{B}}_{3} \mathbf{n}: \nabla \mathbf{u}_{i}-\widehat{\mathfrak{B}}_{1} \widehat{\mathbf{b}} \cdot\left(\nabla \times \mathbf{E}_{H}\right)\right]
$$

with

and

$$
\mathbf{n}: \nabla \mathbf{u}_{i}=\nabla_{\perp} \cdot \mathbf{u}_{\perp i}+\left[A_{\|}, u_{\| i}\right]
$$

or

$$
\mathbf{E}_{H}=\frac{1}{n}\left(\frac{2}{\beta_{e}}(\nabla \times \mathbf{B}) \times \mathbf{B}-\nabla \cdot \mathbf{p}_{e}\right)
$$

Furthermore,

$$
\widehat{\mathbf{b}} \cdot\left(\nabla \times \mathbf{E}_{H}\right)=-\frac{2}{\beta_{e}} \nabla_{\|} \nabla_{\perp}^{2} A_{\|}+\left[n, p_{\perp e}+\frac{2}{\beta_{e}} B_{z}\right] .
$$

$$
\begin{aligned}
& \mathfrak{B}_{1}=\left[\Gamma_{0}\left(b_{i}\right)-\Gamma_{1}\left(b_{i}\right)\right]\left(\frac{\Gamma_{0}\left(b_{i}\right)-\Gamma_{1}\left(b_{i}\right)}{1-\Gamma_{0}\left(b_{i}\right)}+2\right)-\frac{1}{b_{i}} \\
& \mathfrak{B}_{3}=-\mathfrak{B}_{1}-\frac{1}{b_{i}}+\frac{\Gamma_{0}\left(b_{i}\right)-\Gamma_{1}\left(b_{i}\right)}{1-\Gamma_{0}\left(b_{i}\right)} .
\end{aligned}
$$

For $b_{i} \rightarrow 0, \mathfrak{B}_{1} \rightarrow-1 / 4$ and $\mathfrak{B}_{3} \rightarrow-1 / 2$, while for $b_{i} \rightarrow \infty, \mathfrak{B}_{1} \sim-1 / b_{i}$ and $\mathfrak{B}_{3} \sim$ $-1 /\left(2 \sqrt{2 \pi} b_{i}^{3 / 2}\right)$.

At this stage, $\nabla \cdot \mathbf{u}_{i}$, is eliminated by using the continuity equation for the ions

$$
\nabla_{\perp} \cdot \mathbf{u}_{\perp i}=-\partial_{t} n-\left[n, \chi_{s}\right]-\partial_{z} u_{\| i} .
$$

Equation (4.28) becomes

$$
\begin{aligned}
\partial_{t}\left(\omega_{\| i}+\tau \widehat{\mathfrak{B}}_{3} \nabla_{\perp}^{2} n\right)+\left[\omega_{\| i}, \chi_{s}\right]+\tau \widehat{\mathfrak{B}}_{3} \nabla_{\perp}^{2}\left(\left[n, \chi_{s}\right]+\partial_{z} u_{\| i}\right) \\
-\tau\left[n, p_{\perp i}\right]-\left(1-\tau \widehat{\mathfrak{B}}_{1} \nabla_{\perp}^{2}\right)\left[n, p_{\perp e}+\frac{2}{\beta_{e}} B_{z}\right] \\
\quad+\frac{2}{\beta_{e}}\left(1-\tau \widehat{\mathfrak{B}}_{1} \nabla_{\perp}^{2}\right) \nabla_{\|} \nabla_{\perp}^{2} A_{\|}-\tau \widehat{\mathfrak{B}}_{3} \nabla_{\perp}^{2}\left[A_{\|}, u_{\| i}\right]=0 .
\end{aligned}
$$


It is also of interest to write the equation for the ion pressures. Neglecting terms that are subdominant in the present ordering in the case of a scalar equilibrium pressure, the equations for the parallel and perpendicular ion pressure writes

$$
\begin{aligned}
& \frac{D^{(i)}}{D t} p_{\| i}+\nabla \cdot \mathbf{u}_{i}+2 \widehat{\mathbf{b}} \cdot \nabla \mathbf{u}_{i} \cdot \widehat{\mathbf{b}}+\left(\boldsymbol{\Pi} \cdot \nabla \mathbf{u}_{i}\right)^{S}: \boldsymbol{\tau}+\nabla \cdot\left(q_{\| i} \widehat{\mathbf{b}}\right)=0 \\
& \frac{D^{(i)}}{D t} p_{\perp i}+2 \nabla \cdot \mathbf{u}_{i}-\widehat{\mathbf{b}} \cdot \nabla \mathbf{u}_{i} \cdot \widehat{\mathbf{b}}+\left(\boldsymbol{\Pi} \cdot \nabla \mathbf{u}_{i}\right)^{S}: \mathbf{n}+\nabla \cdot\left(q_{\perp i} \widehat{\mathbf{b}}+\mathbf{S}_{\perp i}^{\perp}\right)=0,
\end{aligned}
$$

where the superscript $\mathrm{S}$ indicates that the tensor is symmetrized. The last term in Eq. (4.38) reduces to

$$
\nabla \cdot \mathbf{S}_{\perp i}^{\perp}=\tau \nabla_{\perp}^{2}\left(\widehat{\mathfrak{E}_{4}} \widehat{\mathbf{b}} \cdot\left(\nabla \times \mathbf{E}_{H}\right)+\widehat{\mathfrak{E}_{6}} \mathbf{n}: \nabla \mathbf{u}_{i}\right)
$$

where

$$
\begin{aligned}
\mathfrak{E}_{4}= & {\left[\Gamma_{0}\left(b_{i}\right)-\Gamma_{1}\left(b_{i}\right)\right]\left(\frac{1}{b_{i}}-\frac{\Gamma_{0}\left(b_{i}\right)-\Gamma_{1}\left(b_{i}\right)}{1-\Gamma_{0}\left(b_{i}\right)}\right) } \\
& +2\left(\Gamma_{0}\left(b_{i}\right)-\Gamma_{1}\left(b_{i}\right)\right)-\frac{1}{b_{i}} \Gamma_{1}\left(b_{i}\right) \\
\mathfrak{E}_{6}= & \mathfrak{E}_{4}+\frac{1}{b_{i}}-\frac{\Gamma_{0}\left(b_{i}\right)-\Gamma_{1}\left(b_{i}\right)}{1-\Gamma_{0}\left(b_{i}\right)} .
\end{aligned}
$$

For $b_{i} \rightarrow 0, \mathfrak{E}_{4} \rightarrow 9 / 4$ and $\mathfrak{E}_{6} \rightarrow 3$, while for $b_{i} \rightarrow \infty, \mathfrak{E}_{4} \sim 160 /\left(128 b_{i}^{2} \sqrt{2 \pi b_{i}}\right)$ and $\mathfrak{E}_{6} \sim 1 / b_{i}$. Furthermore, in the present asymptotics, one has for the quantities associated with the work of the nongyrotropic pressure forces

$$
\begin{aligned}
& \left(\boldsymbol{\Pi} \cdot \nabla \mathbf{u}_{i}\right)^{S}: \mathbf{n}=2 \boldsymbol{\Pi}_{\perp}: \nabla_{\perp} \mathbf{u}_{\perp i} \\
& \left(\boldsymbol{\Pi} \cdot \nabla \mathbf{u}_{i}\right)^{S}: \boldsymbol{\tau}=2 \boldsymbol{\Pi}_{\|} \cdot \nabla_{\perp} u_{\| i} .
\end{aligned}
$$

Here $\Pi_{\perp}=\left(\nabla_{\perp} \otimes \nabla_{\perp}-\left(\widehat{\mathbf{z}} \times \nabla_{\perp}\right) \otimes\left(\widehat{\mathbf{z}} \times \nabla_{\perp}\right)\right)\left(-\nabla_{\perp}^{2}\right)^{-1} \mathcal{A}$ and $\Pi_{\|}=-\widehat{\mathbf{b}} \times \nabla \mathcal{D}_{2}$, with $\mathcal{D}_{2}$ given, up to normalization, by Eq. (5.11) of Sulem \& Passot (2015), where the last term is negligible.

The ion temperatures thus obey

$$
\begin{aligned}
& \partial_{t} T_{\| i}+\left[T_{\| i}, \chi_{s}\right]+2 \nabla_{\|} u_{\| i}+\left(\boldsymbol{\Pi} \cdot \nabla \mathbf{u}_{i}\right)^{S}: \boldsymbol{\tau}+\nabla_{\|} q_{\| i}=0, \\
& \partial_{t} T_{\perp i}+\left[T_{\perp i}, \chi_{s}\right]+\left(\boldsymbol{\Pi} \cdot \nabla \mathbf{u}_{i}\right)^{S}: \mathbf{n} \\
& \quad+\nabla_{\|} q_{\perp i}-\widehat{b_{i} \mathfrak{E}_{4}} \widehat{\mathbf{b}} \cdot\left(\nabla \times \mathbf{E}_{H}\right)+\left(1-\widehat{b_{i} \mathfrak{E}_{6}}\right) \mathbf{n}: \nabla \mathbf{u}_{i}=0,
\end{aligned}
$$

where, in the latter equation, one can use Eqs. (4.30) and (4.32). In this equation, $\nabla_{\perp} \cdot \mathbf{u}_{\perp i}$ is again eliminated using the ion continuity equation, which leads to

$$
\begin{aligned}
\partial_{t}( & \left.T_{\perp i}-\left(1-\widehat{b_{i} \mathfrak{E}_{6}}\right) n\right)-\left(1-\widehat{b_{i} \mathfrak{E}_{6}}\right)\left(\left[n, \chi_{s}\right]+\partial_{z} u_{\| i}\right)+\left[T_{\perp i}, \chi_{s}\right]+\left(\boldsymbol{\Pi} \cdot \nabla \mathbf{u}_{i}\right)^{S}: \mathbf{n} \\
& +\nabla_{\|} q_{\perp i}-\widehat{b_{i} \mathfrak{E}_{4}} \widehat{\mathbf{b}} \cdot\left(\nabla \times \mathbf{E}_{H}\right)+\left(1-\widehat{b_{i} \mathfrak{E}_{6}}\right)\left[A_{\|}, u_{\| i}\right]=0 .
\end{aligned}
$$

A closed system (up to the closure of the fluid hierarchy) is thus provided by the dynamical equations (4.6) for $\left(n-B_{z}\right),(4.13)$ for $\left(p_{\| e}-B_{z}\right),(4.14)$ for $\left(p_{\perp e}-2 B_{z}\right)$, (4.16) for $A_{\|}$, (4.20) for $u_{\| i},(4.44)$ for $T_{\| i},(4.46)$ for $\left(T_{\perp i}-\left(1-\widehat{b_{i} \mathfrak{E}_{6}}\right) n\right)$, and (4.36) for $\left(\omega_{\| i}+\tau \widehat{\mathfrak{B}}_{3} \nabla_{\perp}^{2} n\right)$ (with $\omega_{\| i}=-\nabla_{\perp}^{2} \chi_{s}$ ), together with the explicit expressions (4.15) for $u_{\| e}$ and (4.18) for $\varphi$, provided $B_{z}$ given by Eq. (4.25) can be expressed in terms of the 
above quantities. For this purpose, this equation is rewritten

$$
\begin{aligned}
& \left(\tau+2\left(1+\frac{1}{\beta_{e}}\right)+\tau^{2} \widehat{\mathfrak{A}}_{1} \widehat{\mathfrak{B}}_{3} \nabla_{\perp}^{2}+\tau\left(1-\widehat{\mathfrak{A}}_{2}\right)\left(1-\widehat{b_{i} \mathfrak{\mathfrak { E }}_{6}}\right)\right) \delta B_{z}= \\
& \left(\tau+\tau^{2} \widehat{\mathfrak{A}}_{1} \widehat{\mathfrak{B}}_{3} \nabla_{\perp}^{2}+\tau\left(1-\widehat{\mathfrak{A}}_{2}\right)\left(1-\widehat{b_{i} \mathfrak{E}_{6}}\right)\right)\left(\delta n-\delta B_{z}\right)-\left(\delta p_{\perp e}-2 \delta B_{z}\right) \\
& -\tau \widehat{\mathfrak{A}}_{1}\left(\omega_{\| i}+\tau \widehat{\mathfrak{B}}_{3} \nabla_{\perp}^{2} n\right)+\tau\left(1-\widehat{\mathfrak{A}}_{2}\right)\left(\delta T_{\perp i}-\left(1-\widehat{b_{i} \mathfrak{E}_{6}}\right) \delta n\right) .
\end{aligned}
$$

It follows that $B_{z}$ is well-defined, provided

$$
\tau+2\left(1+\frac{1}{\beta_{e}}\right)-\tau b_{i} \mathfrak{A}_{1} \mathfrak{B}_{3}+\tau\left(1-\mathfrak{A}_{2}\right)\left(1-b \mathfrak{E}_{6}\right) \neq 0,
$$

be satisfied, a condition which was numerically checked to hold for any transverse wavenumber.

Appropriate fluid closures can then be chosen in order to express heat fluxes in terms of lower order moments. The simplest assumption is the adiabatic approximation which prescribes zero heat fluxes. A more refined approach is provided by Landau fluid closures (Hammett \& Perkins 1990; Snyder \& Hammett 2001; Sulem \& Passot 2015) which are aimed at reproducing the linear kinetic theory, and thus include Landau damping. Note that in Sec. 6, the heat fluxes are directly computed from the linearized kinetic theory in the low-frequency limit. Involving operators that are non-local in time, this approach is limited to the linearized problem and thus cannot be viewed as a genuine closure.

\section{Asymptotic regimes}

The above model strongly simplifies in two asymptotic regimes which are discussed below. In particular, it will be shown that in such regimes one can retrieve the reduced models derived in Secs. 3.4.1 and 3.4.2 from the gyrofluid approach.

\subsection{The limit of cold ions}

Equations (4.13) and (4.14) are unchanged, while in the limit $\tau \rightarrow 0$, Eq. (4.25) reduces to $p_{\perp e}=-\left(2 / \beta_{e}\right) B_{z}$. Consequently, Eq. (4.6) becomes

$$
\frac{d}{d t}\left(n-B_{z}\right)+\nabla_{\|} u_{\| e}=0
$$

and Eq. (4.18) gives $\chi_{s}=-\varphi$. Furthermore, combining Eq. (4.20) with Eq. (4.16) gives

$$
\frac{d}{d t}\left(u_{\| i}+A_{\|}\right)+\partial_{z} \varphi=0
$$

Using that $\omega_{\| i}=\nabla_{\perp}^{2} \varphi$, Eq. (4.36) reduces to

$$
\frac{d}{d t} \nabla_{\perp}^{2} \varphi+\frac{2}{\beta_{e}} \nabla_{\|} \nabla_{\perp}^{2} A_{\|}=0
$$

Although subdominant, the ion pressures can be evaluated. We first note that, in the limit $\tau \rightarrow 0$, the considered scales are much larger than the ion thermal gyroradius, so one can consider the expression of the nongyrotropic pressure tensor given by Eqs. (3.1)(3.3) of Sulem \& Passot (2015) and derived for example in Schekochihin et al. (2010). In evaluating the contributions of the work of the nongyrotropic pressure forces in the parallel and parallel pressure equations, it is enough to evaluate $\boldsymbol{\Pi}$ to first order, in the form

$$
\boldsymbol{\Pi}=\tau\left(\widehat{\mathbf{b}} \times\left(\nabla \mathbf{u}_{i}\right)^{S} \cdot(\mathbf{I}+3 \boldsymbol{\tau})-(\mathbf{I}+3 \boldsymbol{\tau}) \cdot\left(\nabla \mathbf{u}_{i}\right)^{S} \times \mathbf{b}\right) .
$$


One easily checks that in this case, $\left(\boldsymbol{\Pi} \cdot \nabla \mathbf{u}_{i}\right)^{S}: \mathbf{n}$ and $\left(\boldsymbol{\Pi} \cdot \nabla \mathbf{u}_{i}\right)^{S}: \boldsymbol{\tau}$ are subdominant and can be neglected.

Combining Eq. (4.44) with Eq. (5.1), and using Eq. (4.15) together with Eq. (5.3), one gets

$$
\frac{d}{d t}\left(p_{\| i}-B_{z}-\nabla_{\perp}^{2} \varphi\right)+\nabla_{\|}\left(3 u_{\| i}+q_{\| i}\right)=0 .
$$

Similarly, from Eqs. (4.46) and (4.6), it follows that

$$
\frac{d}{d t}\left(p_{\perp i}-2 B_{z}-2 \nabla_{\perp}^{2} \varphi\right)+\nabla_{\|}\left(u_{\| i}+q_{\perp i}\right)=0 .
$$

To summarize, in the cold-ion asymptotic regime, the reduced model derived from the FLR-Landau fluid yields the model of Sec. 3.4.1. Indeed, the electron equations (5.1), (4.16), (4.13), (4.14) correspond namely to Eqs. (3.52), (3.53), (3.54) and (3.55), respectively. Similarly, the ion equations (5.2), (5.5) and (5.6), correspond to Eqs. (3.57), (3.58) and (3.59), respectively. The vorticity equation (5.3), on the other hand, together with the quasi-neutrality assumption $n_{e}=n_{i}$, which is intrinsic to the FLR reduced fluid model, and with the electron continuity equation (5.1), yields Eq. (3.56). Finally, Eq. (4.15) corresponds to Eq. (3.61) and, as above stated, Eq. (4.25) reduces to Eq. (3.62).

Note that when assuming a polytropic scalar electron pressure, the present cold-ion model reduces to the Hamiltonian four-field model of Tassi et al. (2010) (when neglecting electron inertia), which extends to three dimensions the two-dimensional model of Fitzpatrick \& Porcelli $(2004,2007)$.

\subsection{The sub-ion scale limit}

The equations for the electrons remain valid in this regime, and the main issue concerns the ion dynamics. In particular, it will be shown that ion velocities are subdominant and, as a consequence, the perpendicular ion pressure satisfies an isothermal equation of state. In order to address this issue, the additional small parameter $\mu \propto k_{\perp}^{-1}$ measuring the magnitude of the inverse transverse wavenumber must be introduced. While the smallness of the parameter $\mu$ is physically limited by the ratio of the electron to ion gyroradius, and thus by the square root of the electron to ion mass ratio, it is technically relevant to take the limit $\mu \rightarrow 0$ where important simplifications take place. In particular, the operators $\left(1-\widehat{b_{i} \mathfrak{A}_{1}}\right),\left(1+\widehat{b_{i} \mathfrak{B}_{1}}\right)$ and $\widehat{b_{i} \mathfrak{E}_{4}}$ vanish asymptotically, while $\widehat{b_{i} \mathfrak{E}_{6}}=1$ and ${\widehat{b_{i} \mathfrak{B}_{3}}}_{3}=-1 /\left(2 \sqrt{2 \pi} b_{i}^{3 / 2}\right)$.

The amplitude of the fields $\delta n, \varphi, \delta B_{z}, \delta p_{\perp e}$ and $\delta p_{\| e}$ are now scaled by the small parameter $\epsilon \mu$. The parallel space variable is stretched by a factor $\epsilon$, while the time variable is scaled by the factor $\epsilon / \mu$, that results from a dominant balance between the linear terms of the electronic equations. This regime is indeed dominated by the dynamics of kinetic Alfvén waves (KAWs) whose dispersion relation is $\omega \propto k_{\|} k_{\perp}$.

A first remark is that in the material derivative, the transport operator $\left[\cdot, \chi_{s}\right]=$ $\left(1 / \mu^{2}\right) \mathcal{O}\left(\chi_{s}\right)$ is negligible compared to $\partial_{t}=\mathcal{O}(\epsilon / \mu)$. Indeed, otherwise, $\chi_{s}$ would be larger than $\epsilon \mu$ and thus $\mathbf{u}_{\perp i}$ larger than $\epsilon$, which is unrealistic in the present ordering. This point will be checked a posteriori.

From Eq. (4.16), it follows that $A_{\|}=\mathcal{O}\left(\epsilon \mu^{2}\right)$, and from Eq. (4.15) that $u_{\| e}=\mathcal{O}(\epsilon)$, provided the magnitude of $u_{\| i}$ does not exceed $\epsilon$. From the density equation, $\nabla \cdot \mathbf{u}_{i}=$ $\mathcal{O}\left(\epsilon^{2}\right)$, and so is the transverse part $\nabla_{\perp} \cdot \mathbf{u}_{\perp i}$.

Equation (4.28) then reduces to

$$
\frac{\partial}{\partial t} \omega_{\| i}-\tau\left[n, p_{\perp i}\right]-1 /\left(\widehat{\left(2 \sqrt{2 \pi} b_{i}\right.}\right)\left(\mathbf{n}: \nabla \mathbf{u}_{i}\right)=0 .
$$


The last term is $\mathcal{O}\left(\epsilon^{2} \mu\right)$. It follows that, provided $\delta T_{\perp i} \lesssim \mathcal{O}\left(\epsilon \mu^{2}\right)$, one has $\chi_{s}=\mathcal{O}\left(\epsilon \mu^{4}\right)$ and $\left|\mathbf{u}_{\perp i}\right|=\mathcal{O}\left(\epsilon \mu^{3}\right)$, consistent with the previous assumptions.

Let us now turn to the equation for the ion perpendicular pressure. As in this limit, $\boldsymbol{\Pi}_{\perp}$ involves only the temperature contribution, $\left(\boldsymbol{\Pi} \cdot \nabla \mathbf{u}_{i}\right)^{S}: \mathbf{n}=\mathcal{O}(\epsilon \mu) \mathcal{O}\left(\delta T_{\perp i}\right)$ and is thus negligible in Eq. (4.45) which reduces to

$$
\frac{\partial T_{\perp i}}{\partial t}+\nabla_{\|} q_{\perp i}=\mathcal{O}\left(\epsilon^{2} \mu\right)
$$

The latter equation describes the relaxation of the parallel temperature fluctuations under the effect of the heat flux, leading indeed to $\delta T_{\perp i}=\mathcal{O}\left(\epsilon \mu^{2}\right)$.

Returning to Eq. (4.26), we find that the fluctuations $\delta \varphi$ of $\varphi$ and $\delta n$ of $n$ satisfy

$$
\delta \varphi=-\tau \delta n
$$

which is Eq. (3.76). The term $\mathcal{A}$ in the pressure balance equation (4.22) is also subdominant and condition (3.78) is recovered.

The last point concerns Eq. (3.77), which requires that the ion parallel velocity be smaller than $\epsilon$. In the small-scale limit, the electron pressure gradient terms cancel out in Eq. (4.20). Using Eq. (5.9), this equation reduces to

$$
\partial_{t} u_{\| i}+\nabla_{\|} T_{\| i}=\mathcal{O}\left(\epsilon^{2} \mu^{2}\right),
$$

where the driving term in the rhs originates from the subdominant contribution to $\mathfrak{C}_{2}$.

Furthermore, in the equation for the parallel pressure, one has to evaluate the work of the nongyrotropic pressure given by $2 \Pi_{\|} \nabla_{\perp} \cdot u_{\| i}$ where in the small-scale limit $\Pi_{\|}=$ $-\widehat{\mathbf{b}} \times \nabla_{\perp} \mathcal{D}_{2}$. The evaluation of this term requires considering the asymptotic behavior for $b_{i} \rightarrow \infty$ of the various coefficients constructed with the functions $\Gamma_{0}$ and $\Gamma_{1}$ in the expression of $\mathcal{D}_{2}$. It turns out that $\mathcal{D}_{2}$ scales like $\epsilon \mu^{2}$, so $\boldsymbol{\Pi}_{\|}=\mathcal{O}(\epsilon \mu)$ and $2 \boldsymbol{\Pi}_{\|} \cdot \nabla_{\perp} u_{\| i}=$ $\mathcal{O}\left(\epsilon^{2} \mu^{2}\right) \mathcal{O}\left(\left|u_{\|}\right|\right)$. This term is asymptotically small compared to $\nabla_{\|} u_{\| i}$. The equation for the ion parallel temperature thus reduces to

$$
\partial_{t} T_{\| i}+2 \nabla_{\|} u_{\| i}+\partial_{\|} q_{\| i}=0
$$

Without specifying the heat flux $q_{\|}$, it is possible to derive magnitude estimates from Eqs. (5.10) and (5.11). The latter equation can be viewed as describing the relaxation of the parallel temperature fluctuations under the effect of the heat flux, in the presence of a driving originating from the parallel velocity fluctuations. This suggests that the first term in Eq. (5.11) cannot exceed the second one, indicating that the magnitude of the ratio $T_{\| i} / u_{\| i}$ cannot exceed $\mu$. This makes the temperature contribution negligible in Eq. (5.10), which ensures that $u_{\| i}=\mathcal{O}\left(\epsilon \mu^{3}\right)$. Consequently, $T_{\| i} \leqslant \mathcal{O}\left(\epsilon \mu^{4}\right)$. It follows that both the ion temperature fluctuations and the ion parallel velocity are subdominant and can be neglected in this regime.

Interestingly, the above estimates are consistent with the linear kinetic theory. Indeed, inspection of Eqs. (B5) and (B7) of Passot \& Sulem (2007) indicates that, at the level of the linear kinetic theory,

$$
\frac{T_{\| i}}{u_{\| i}}=\frac{1}{\sqrt{2 \tau}} \frac{1-R\left(\zeta_{i}\right)+2 \zeta_{i}^{2} R\left(\zeta_{i}\right)}{\zeta_{i} R\left(\zeta_{i}\right)},
$$

where $R$ is the plasma response function related to the plasma dispersion function $Z$ by $R(\zeta)=1+\zeta Z(\zeta)$, and $\zeta_{i}=(1 / \sqrt{2 \tau})\left(\omega /\left|k_{z}\right|\right)$. It follows that for large $\zeta_{i}, T_{\| i} / u_{\| i}=$ $\mathcal{O}\left(1 / \zeta_{i}\right)=\mathcal{O}(\mu)$. 
Similarly, at the level of the linear kinetic theory, one also has,

$$
\frac{q_{\| i}}{T_{\| i}}=\sqrt{2 \tau} \frac{\zeta_{i}\left[1-3 R\left(\zeta_{i}\right)+2 \zeta_{i}^{2} R\left(\zeta_{i}\right)\right]}{1-R\left(\zeta_{i}\right)+2 \zeta_{i}^{2} R\left(\zeta_{i}\right)},
$$

which behaves like $3 /\left(2 \zeta_{i}\right)$ when $\zeta_{i} \rightarrow \infty$, indicating that $q_{\| i}$ is subdominant in Eq. (5.11). The two other terms balance each other, consistent with $T_{\| i} / u_{\| i}=\mathcal{O}(\mu)$.

The complete system of equations of the small-scale gyrofluid model can thus be recovered by the FLR-Landau fluid approach in the limit of small amplitude and large transverse wavenumbers. Indeed, as previously mentioned, the electron equations (4.6), (4.16), (4.13), (4.14) yield Eqs. (3.72), (3.73), (3.74) and (3.75), respectively. Eq. (5.9) corresponds to Eq. (3.76). Finally, the above arguments leading to isotropic, isothermal ion fluid, with negligible parallel velocity, yield Eqs. (3.77) and (3.78) from Eqs. (4.15) and (4.18), respectively.

Note that the order of magnitude estimates for the various fields (and in particular for the ion vorticity and divergence) from the fluid system are consistent with the kinetic low frequency linear formulae of Appendix B in Passot \& Sulem (2007). The non-trivial cancellations with FLR corrections mentioned above are crucial to obtain these estimates.

\section{Validity range of the small-scale gyrofluid model}

It is of interest to study the validity of the small-scale model by analyzing, in the linear regime, its prediction for the dispersion and damping of kinetic Afvén waves. For this purpose, and in order not to introduce additional uncertainty related to the hierarchy closure, electron heat fluxes are evaluated from the low-frequency kinetic theory, as done in Passot \& Sulem (2007). Indeed, in the present framework where the electron FLR corrections are neglected, Eqs. (B12) and (B7) of this reference give

$$
q_{\| e}=\sqrt{\frac{2 M}{m}} \frac{\zeta_{e}\left[1-3 R\left(\zeta_{e}\right)+2 \zeta_{e}^{2} R\left(\zeta_{e}\right)\right]}{1-R\left(\zeta_{e}\right)+2 \zeta_{e}^{2} R\left(\zeta_{e}\right)} \delta T_{\| e} .
$$

Here $\zeta_{e}=(1 / \sqrt{2 M / m})\left(\omega /\left|k_{z}\right|\right)$ (where $\omega$ is the complex frequency) and $R$ is the plasma response function $R\left(\zeta_{e}\right)=1+\zeta_{e} Z\left(\zeta_{e}\right)$, with $Z$ denoting the plasma dispersion function. Similarly, Eqs. (B13) and (B6) lead to

$$
q_{\perp e}=-\sqrt{\frac{2 M}{m}} \frac{\zeta_{e} R\left(\zeta_{e}\right)}{1-R\left(\zeta_{e}\right)} \delta T_{\perp e} .
$$

In order to simplify the writing, it is convenient to use the notations $q_{\| e}=\mathcal{Q}_{\|}\left(\zeta_{e}\right) \delta T_{\| e}$ and $q_{\perp e}=\mathcal{Q}_{\perp}\left(\zeta_{e}\right) \delta T_{\perp e}$. The linear system reads (keeping the same notation for the fields and their Fourier modes)

$$
\begin{aligned}
& \omega\left(\left(1+\frac{\tau \beta_{e}}{2}\right) \delta n+\frac{\beta_{e}}{2} \delta p_{\perp e}\right)+\frac{2 k_{z} k_{\perp}^{2}}{\beta_{e}} A_{\|}=0 \\
& \omega A_{\|}+k_{z}\left(\tau \delta n+\delta p_{\| e}\right)=0 \\
& \omega\left(\frac{\tau \beta_{e}}{2} \delta n+\delta p_{\| e}+\frac{\beta_{e}}{2} \delta p_{\perp e}\right)+k_{z}\left(-\mathcal{Q}_{\|}\left(\zeta_{e}\right)\left(\delta p_{\| e}-\delta n\right)+\frac{6 k_{\perp}^{2}}{\beta_{e}} A_{\|}\right)=0 \\
& \omega\left(\tau \beta_{e} \delta n+\left(1+\beta_{e}\right) \delta p_{\perp e}\right)+k_{z}\left(-\mathcal{Q}\left(\zeta_{e}\right)\left(\delta p_{\perp e}-\delta n\right)+\frac{2 k_{\perp}^{2}}{\beta_{e}} A_{\|}\right)=0 .
\end{aligned}
$$

It is easily seen that the parallel wavenumber only enters in the combination $\omega / k_{z}$. 
Equations (6.3)-(6.6) are conveniently solved numerically. In order to test the validity of this gyrofluid model, we also solve for the linear fully kinetic solution using the WHAMP software (Rönnmark 1982), and for a low-frequency limit, equivalent to the gyrokinetic approach. It consists in substituting the kinetic expressions of the ion and electron density and velocity, as given in Appendix B of Passot \& Sulem (2007), within the quasi-neutrality condition, the parallel component of the Ampère equation and of its curl (which isolates the contribution of the solenoidal velocities). This leads to a linear system of three equations. The dispersion relation is then obtained by solving for the determinant of a $3 \times 3$ matrix $\mathfrak{M}$ whose elements (in the case of isotropic pressures) are given by (Kuznetsov et al. 2012)

$$
\begin{aligned}
\mathfrak{M}_{11}= & -\left(\Gamma_{0}\left(b_{i}\right)-\Gamma_{1}\left(b_{i}\right)\right)\left(R\left(\zeta_{i}\right)-1\right)+\left(\Gamma_{0}\left(b_{e}\right)-\Gamma_{1}\left(b_{e}\right)\right)\left(R\left(\zeta_{e}\right)-1\right) \\
\mathfrak{M}_{12}= & \Gamma_{0}\left(b_{i}\right)-1+\tau\left(\Gamma_{0}\left(b_{e}\right)-1\right) \\
\mathfrak{M}_{13}= & \left.-\left[\Gamma_{0}\left(b_{i}\right) R\left(\zeta_{i}\right)+1-\Gamma_{0}\left(b_{i}\right)\right]-\tau\left[\Gamma_{0}\left(b_{e}\right) R\left(\zeta_{e}\right)+1-\Gamma_{0}\left(b_{e}\right)\right)\right] \\
\mathfrak{M}_{21}= & \left(\Gamma_{1}\left(b_{i}\right)-\Gamma_{0}\left(b_{i}\right)\right) R\left(\zeta_{i}\right)-\left(\Gamma_{1}\left(b_{e}\right)-\Gamma_{0}\left(b_{e}\right)\right) R\left(\zeta_{e}\right) \\
\mathfrak{M}_{22}= & -\frac{k_{\perp}^{2}}{\beta_{e} \zeta_{i}^{2}} \\
\mathfrak{M}_{23}= & -\left[\Gamma_{0}\left(b_{i}\right) R\left(\zeta_{i}\right)+\tau \Gamma_{0}\left(b_{e}\right) R\left(\zeta_{e}\right)\right] \\
\mathfrak{M}_{31}= & \left.\tau \beta_{e}\left(\Gamma_{0}\left(b_{i}\right)-\Gamma_{1}\left(b_{i}\right)\right)\left(R\left(\zeta_{i}\right)-1\right)+\beta_{e}\left(\Gamma_{0}\left(b_{e}\right)-\Gamma_{1}\left(b_{e}\right)\right)\left(R\left(\zeta_{e}\right)\right)-1\right) \\
& -1-\frac{k_{z}^{2}}{k_{\perp}^{2}} \\
\mathfrak{M}_{32}= & -\frac{\tau \beta_{e}}{2}\left(\Gamma_{0}\left(b_{i}\right)-\Gamma_{1}\left(b_{i}\right)-\Gamma_{0}\left(b_{e}\right)+\Gamma_{1}\left(b_{e}\right)\right) \\
\mathfrak{M}_{33}= & \frac{\tau \beta_{e}}{2}\left[\left(\Gamma_{0}\left(b_{i}\right)-\Gamma_{1}\left(b_{i}\right)\right)\left(R\left(\zeta_{i}\right)-1\right)\right. \\
& \left.\left.-\left(\Gamma_{0}\left(b_{e}\right)-\Gamma_{1}\left(b_{e}\right)\right)\left(R\left(\zeta_{e}\right)\right)-1\right)\right]
\end{aligned}
$$

where $b_{\alpha}=\left(k_{\perp}^{2} \rho_{\alpha}^{2}\right) / 2$, with $\alpha=i$ for the ions and $\alpha=e$ for the electrons. In the latter approach, two cases can be distinguished, depending on whether the electron FLR effects are retained or neglected (by taking $b_{e}=0$ ). In all the three considered kinetic approaches, electron inertia is implicitly built in, while it is neglected in the small-scale gyrofluid model and thus in Eqs. (6.3)-(6.6).

In the linear regime, the angle of propagation $\theta$ between the ambient field and the wavevector has to be specified. While it has almost no influence in the low-frequency asymptotic description, it has to be chosen close to $90^{\circ}$ in the Vlasov description in order to avoid resonances. We have taken $\theta=89.9^{\circ}$ in order to prevent any finite angle dependence in the considered range of wavenumbers). We further assume $\beta_{e}=\tau=1$.

For comparison, we also consider the adiabatic and isothermal closures. In the adiabatic case $\left(q_{\| e}=q_{\perp e}=0\right)$, an analytic solution is found for the frequency in the form

$$
\frac{\omega^{2}}{k_{z}^{2} k_{\perp}^{2}}=\frac{4 \tau+12+(6 \tau+10) \beta_{e}}{\beta_{e}\left[2+(\tau+2) \beta_{e}\right]} .
$$

This solution contrasts from the isothermal one

$$
\frac{\omega^{2}}{k_{z}^{2} k_{\perp}^{2}}=\frac{2(1+\tau)}{\beta_{e}\left[1+(1+\tau) \beta_{e} / 2\right]},
$$


obtained by solving the subsystem of equations for $\delta n_{e}$ and $A_{\|}$, when imposing $\delta p_{\| e}=$ $\delta p_{\perp e}=\delta n$. In this regime, following Howes et al. (2006), an estimate of the Landau damping can be obtained in the form

$$
\frac{\gamma}{k_{z} k_{\perp}^{2}}=-\frac{2 \pi m}{\beta_{e} M}\left(1-\frac{1+(1+\tau) \beta_{e}}{2\left[1+(1+\tau) \beta_{e} / 2\right]^{2}}\right) .
$$

Figure 1 displays the real and imaginary parts $\Re(\omega) / k_{z}$ (left) and $\Im(\omega) / k_{z}$ (right) of the complex frequency as a function of $k_{\perp}$, resulting from the various approximations described above. The solid lines correspond to the fully kinetic system. The lowfrequency kinetic description, for which the dispersion relation is given by the the con$\operatorname{dition} \operatorname{det}(\mathfrak{M})=0$, is considered in two different cases. In the first one (referred to by + symbols), no assumptions are made on $b_{i}$ and $b_{e}$ but for numerical convenience we take $R\left(\zeta_{i}\right)=0$ which is valid for $k_{\perp}$ larger than a few units, as expected for small-scale kinetic Alfvén waves for which $\omega / k_{z} \sim k_{\perp} \gg 1$. Furthermore, $R\left(\zeta_{e}\right)$ is approximated by a 4 -pole Padé approximant with the parameters $(N, p)$ defined in Table 1 of Hedrick \& Leboeuf (1992), are taken equal to $(4,1)$, for which the plasma response function is globally approximated with a good accuracy. In the second case (referred to by $*$ symbols), we concentrate on the small-scale asymptotic regime corresponding in the present notations to $b_{e}=0$ and $b_{i}=\infty$ (here taken equal to $10^{9}$ ). In this setting, the functions $R\left(\zeta_{i}\right)$ and $R\left(\zeta_{e}\right)$ are both approximated by the above 4-pole Padé approximant. The reduced model given by Eqs.(6.1)-(6.6) is indicated by $\diamond$ symbols. Finally, the upper (lower) dashed lines correspond to the adiabatic (isothermal) solutions, the dashed line of Fig. 1 (right) corresponding to the isothermal analytic damping rate, as given by Eq. (6.18).

It is to be noticed that for the considered large angle of propagation, the low-frequency limit with ions and electrons FLR corrections (+ symbols) displays almost no deviation with respect to the fully kinetic solution up to $k_{\perp}=30$ (in $\rho_{s}^{-1}$ units), confirming the validity of the small-frequency asymptotics. When neglecting electron FLR correction in the low-frequency kinetic theory ( $*$ symbols), some inaccuracy starts to be visible on the real frequency (but not on the damping rate) by $k_{\perp}=15$, but remains moderate for all the considered scales. Differently, the gyrofluid ( $\diamond$ symbols) precisely predicts the real frequency and the damping rate at moderately small scales but significantly departs from the kinetic theory when $k_{\perp}>15$, suggesting the importance of electron inertia (neglected in the present theory) for $k_{\perp} \rho_{i} \gtrsim 20$. When comparing with the real frequency predicted by the conservative closures, we note that, while the adiabatic approximation is inaccurate at all the scales, assuming isothermal electron provides a rather good approximation of the real frequency up to the electron gyroscale.

\section{Conclusion}

We have considered a three-dimensional gyrofluid model accounting for magnetic fluctuations along the guide field in two asymptotic regimes. One concerns the limit of cold ions, the other concentrates on the spectral domain corresponding to transverse scales large compared to the electron gyroradius but small relatively to the ion gyroradius. The cold-ion model extends the "Hall reduced MHD" (Schekochihin et al. 2009), by including a realistic description of anisotropic electron temperatures. The spectral validity range of the second model may seem limited, as the ratio of the ion to electron thermal gyroradii is given by the square root of the particle mass ratio. This model nevertheless deserves a special attention, as it extends the kinetic Alfvén wave model of Boldyrev et al. (2013), by retaining dynamical equations for the electron gyrotropic pressures instead of assuming 

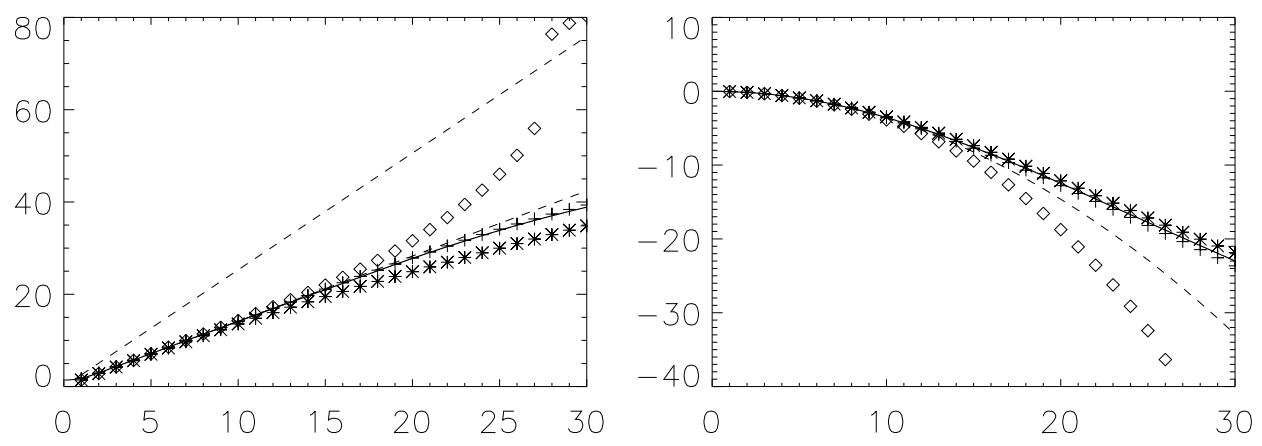

FigURE 1. Real part (left panel) and imaginary part (right panel) of $\omega / k_{z}$ versus $k_{\perp}$ (in units of $\left.\rho_{s}^{-1}\right)$ for KAWs, in the conditions of the small-scale model, within various descriptions: adiabatic and isothermal regimes (upper and lower dashed lines respectively, in the left panel) together with the corresponding analytic damping rate in the isothermal case (dashed in the right panel), gyrofluid model (diamonds), full kinetic theory assuming $\theta=89.9^{\circ}$ (solid line), low-frequency kinetic theory including ions and electrons FLR corrections (crosses) and without electron FLR contributions (stars).

an isothermal electron fluid. Interestingly, this limit involves important simplifications, such as subdominant ion velocity and temperature fluctuations.

In both models, the pressure equations involve electron heat fluxes and energy-weighted pressures (fourth order cumulants) which are to be estimated by prescribing closure relations, possibly involving Landau damping (see Sulem \& Passot (2015) and references therein), an issue outside the scope of the present paper.

It turns out that the two asymptotic gyrofluid models can also be viewed as suitable weakly-nonlinear limits of the FLR-Landau fluid (Sulem \& Passot 2015) where ion nongyrotropic (i.e. FLR) contributions are estimated from the low-frequency linear kinetic theory phenomenologically corrected to take magnetic field line distortions into account. Although this approach is less systematic that the gyrofluid formalism, it provides an easier identification of the physical origin of the various terms arising in the model equations, such as the electron energy-weighted pressures in the pressure equations. Also, the fact that ion velocity and temperature fluctuations are negligible in the small-scale model results in the fluid description from the cancellation of some hydrodynamic terms by FLR contributions. The agreement with the gyrofluid indicates that, at least in the case of isotropic equilibrium pressures, the FLR-Landau fluid built to fit the linear kinetic theory is also quantitatively accurate in the weakly-nonlinear regime addressed by the gyrokinetic formalism.

As the second model is limited to scales large compared to the electron gyroradius, we evaluated more quantitatively its spectral validity range, by making comparisons at the level of dispersion and damping of kinetic Alfvén waves between the full linear kinetic theory as provided by the WHAMP software (Rönnmark 1982) and the small-scale model where the heat fluxes and fourth-order cumulants (not given by the model) are evaluated asymptotically from the low-frequency kinetic theory, as in Appendix B of Passot \& Sulem (2007). It turns out that for isotropic and equal ion and electron temperatures and corresponding betas equal to unity, a good agreement holds for $k_{\perp} \rho_{e} \lesssim 20$.

Further developments include the extension of the small-scale model to electron scales. Assuming for the sake of simplicity small betas for which nongyrotropic pressure corrections are negligible, leading order corrections originating from electron inertia are to be retained. Such an effect would in particular be needed to simulate collisionless magnetic 
reconnection. Furthermore, in the derivation of the asymptotic model from the FLRLandau fluid, an intermediate step led to a reduced formulation of the FLR-Landau fluid valid at scales comparable to the ion gyroradius, in the weakly-nonlinear regime when the equilibrium temperatures are isotropic. In this regime, the ion dynamics is relevant and the corresponding model could be useful for a detailed analysis of the role of the ion Landau damping which is supposed to potentially affect the kinetic Alfvén wave cascade (Passot \& Sulem 2015; Sulem et al. 2016).

\section{REFERENCES}

Boldyrev, S., Horaites, K., XiA, Q. \& Perez, J. C. 2013 Toward a theory of astrophysical plasma turbulence at subproton scales. Astrophys. J. 777, 41.

BrizARD, A. 1989 Nonlinear gyrokinetic Maxwell-Vlasov equations using magnetic co-ordinates. J. Plasma Phys. 41, 541-559.

Brizard, A. 1992 Nonlinear gyrofluid description of turbulent magnetized plasmas. Phys. Fluids 4, 1213-1228.

Dorland, W. \& Hammett, G. W. 1993 Gyrofluid turbulence models with kinetic effects. Phys. Fluids B 5, 812-835.

FitzPatrick, R. \& Porcelli, F. 2004 Collisionless magnetic reconnection with arbitrary guide field. Phys. Plasmas 11, 4713-4718.

Fitzpatrick, R. \& Porcelli, F. 2007 Collisionless magnetic reconnection with arbitrary guide field (erratum). Phys. Plasmas 14, 049902.

Hammett, G. W. \& Perkins, F. W. 1990 Fluid moment models for Landau damping with application to the ion-temperature gradient instability. Phys. Rev. Lett 64, 3019-3022.

Hazeltine, R. D., Hsu, C. T. \& Morrison, P. J. 1987 Hamiltonian four-field model for nonlinear tokamak dynamics. Phys. Fluids 30, 3204-3211.

Hazeltine, R. D., Kotschenreuther, K. \& Morrison, P. J. 1985 A four-field model for tokamak plasma dynamics. Phys. Fluids 28, 2466-2477.

Hedrick, C. L. \& Leboeuf, J. N. 1992 Landau fluid equations for electromagnetic and electrostatic fluctuations. Phys. Plasmas B4, 3915-3934.

Howes, G. G., Cowley, S. C., Dorland, W., Hammett, G. W., E.Quataert \& Scherochinin, A. A. 2006 Astrophysical gyrokinetics: basic equations and linear theory. Astrophys. J. 651, 590-614.

Hsu, C. T., Hazeltine, R. D. \& Morrison, P. J. 1986 A generalized reduced fluid model with finite ion-gyroradius effects. Phys. Fluids 29, 1480-1487.

Kiyani, K. H., Chapman, S. C., Sahraoui, F., Hnat, B., Fauvarque, O. \& Khotyaintsev, YU. V. 2013 Enhanced magnetic compressibility and isotropic scale invariance at sub-ion Larmor scales in solar wind turbulence. Astrophys. J. 663, 10.

Kuznetsov, E., Passot, T. \& Sulem, P. L. 2012 On the mirror instability in the presence of electron temperature anisotropy. J. Plasma Phys. 19, 092116.

Morrison, P. J. 1998 Hamiltonian description of the ideal fluid. Rev. Mod. Phys. 70, 467-521.

Passot, T. \& Sulem, P. L. 2007 Collisionless magnetohydrodynamics with gyrokinetic effects. Phys. Plasmas 14, 082502.

Passot, T. \& Sulem, P. L. 2015 A model for the non-universal power-law of the solar wind sub-ion scale magnetic spectrum. Astrophys. J. Lett. 812, L37.

RÖNNMARK, K. 1982 Waves in homogeneous, anisotropic multicomponent plasmas (WHAMP). Tech. Rep. 179. Kiruna Geophysical Institute.

Scherochinin, A. A., Cowley, S. C., Rincon, F. \& Rosin, M. S. 2010 Magnetofuid dynamics of magnetized cosmic plasma: firehose and gyrothermal instabilities. Mon. Not. R. Astron. Soc. 405, 291-300.

Schekochinin, A. A., Cowley, S. C., W. Dorland, G. W. Hammett, Howes, G. G., Quataert, E. \& TAtsuno, T. 2009 Astrophysical gyrokinetics: kinetic and fluid turbulent cascades in magnetized weakly collisional plasmas. Astrophys. J. Supp. 182, 310.

ScotT, B.D. 2010 Derivation via free energy conservation constraints of gyrofluid equations with finite-gyroradius electromagnetic nonlinearities. Phys. Plasmas 17, 102306. 
Snyder, P. B. \& Hammett, G. W. 2001 A Landau fluid model for electromagnetic plasma microturbulence. Phys. Plasmas 8, 3199-3216.

Sulem, P. L \& PAssot, T. 2015 Landau fluid closures with nonlinear large-scale finite Larmor radius corrections for colliionless plasmas. J. Plasma Phys. $\mathbf{8 1}$.

Sulem, P. L., Passot, T., Laveder, D. \& Borgogno, D. 2016 Influence of the nonlinearity parameter on the solar-wind sub-ion magnetic energy spectrum: FLR-Landau fluid simulations. Astrophys. J. 818.

Tassi, E., Morrison, P.J., Grasso, D. \& Pegoraro, F. 2010 Hamiltonian four-field model for magnetic reconnection: nonlinear dynamics and extension to three dimensions with externally applied fields. Nucl. Fusion 50, 034007.

Thiffeault, J. L. \& Morrison, P. J. 2000 Classification and Casimir invariants of Lie-Poisson brackets. Physica D 136, 205-244.

Waelbroeck, F.L. \& TAssi, E. 2012 A compressible Hamiltonian electromagnetic gyrofluid model. Commun. Nonlinear Sci. Numer. Simulat. 17, 2171. 